\title{
Collider searches for scalar singlets across lifetimes
}

\author{
Elina Fuchs, ${ }^{a, b}$ Oleksii Matsedonskyi, ${ }^{c}$ Inbar Savoray ${ }^{c}$ and Matthias Schlaffer ${ }^{a}$ \\ ${ }^{a}$ University of Chicago, Department of Physics, \\ 5720 South Ellis Avenue, Chicago, IL 60637 U.S.A. \\ ${ }^{b}$ Fermilab, Theory Department, \\ Kirk Road and Pine Street, Batavia, IL 60510, U.S.A. \\ ${ }^{c}$ Weizmann Institute of Science, \\ 234 Herzl Street, Rehovot 7610001, Israel \\ E-mail: elinafuchs@uchicago.edu, oleksii.matsedonskyi@weizmann.ac.il, \\ Inbar.Savoray@weizmann.ac.il, Schlaffer@uchicago.edu
}

ABSTRACT: Spin-0 singlets arise in well-motivated extensions of the Standard Model. Their lifetime determines the best search strategies at hadron and lepton colliders. To cover a large range of singlet decay lengths, we investigate bounds from Higgs decays into a pair of singlets, considering signatures of invisible decays, displaced and delayed jets, and coupling fits of untagged decays. We examine the generic scalar singlet and the relaxion, and derive a matching as well as qualitative differences between them. For each model, we discuss its natural parameter space and the searches probing it.

Keywords: Beyond Standard Model, Higgs Physics

ARXiv EPrint: 2008.12773 


\section{Contents}

1 Introduction 1

2 Singlet extension of the Higgs sector 2

2.1 Renormalizable singlet 4

2.2 Relaxion 5

2.2.1 Comparison to singlet extension 6

$\begin{array}{lll}\text { 2.2.2 Theoretical bounds on the parameter space } & 7\end{array}$

3 Collider bounds on (long-lived) scalar singlets $\quad 8$

3.1 Fits of untagged and invisible Higgs decays $\quad 9$

$\begin{array}{lll}3.2 & \text { Displaced jets } & 13\end{array}$

3.3 Delayed jets 14

$\begin{array}{lll}3.4 & \text { Searches for invisible Higgs decays } & 15\end{array}$

$\begin{array}{lll}4 & \text { Overview } & 17\end{array}$

5 Conclusions 21

$\begin{array}{ll}\text { A Relaxion stopping point } & 23\end{array}$

B Estimating singlet production via Higgs mixing 25

C Timing of delayed jets 25

\section{Introduction}

Light spin-zero singlets are ubiquitous in models of New Physics (NP). They can have important phenomenological roles such as serving as a portal to a Dark Sector [1] and rendering the electroweak phase transition first order to enable electroweak baryogenesis $[2$, 3]. For recent studies of the compatibility of a light singlet with a strong electroweak phase transition and Higgs properties, see e.g. refs. [4, 5]. In many cases, the phenomenology associated with such NP can be encompassed in the minimal renormalizable extension of the Standard Model (SM) obtained by adding one spin-zero singlet $\phi$ [6]. We consider this model as a benchmark, assuming all other new degrees of freedom are sufficiently heavy or weakly coupled to the SM particles (for effects of dimension-five operators in the singlet extension, see e.g. [7]).

Despite its simple setup, the singlet extension brings about a rich phenomenology related to the Higgs, by opening the exotic decay channel $h \rightarrow \phi \phi$, if kinematically allowed (see e.g. ref. [8]), and by reducing the couplings of the Higgs boson to SM particles via singlet-Higgs mixing. This applies equally to scalars and pseudoscalars, though in the latter case the $\phi$-Higgs mixing requires breaking of $\mathcal{C P}$. The phenomenological implications reach 
far beyond Higgs-related observables, as the singlet inherits the couplings of the Higgs to the SM particles, suppressed by the mixing angle. Therefore, the interactions of the singlet can, depending on its mass $m_{\phi}$, be probed across the precision, luminosity and energy frontiers. The various signatures of the singlet include its effects on atomic physics, tests of the equivalence principle, Dark Matter (DM), beam dump experiments, rare meson decays, collider signatures as well as astrophysical and cosmological observables, see e.g. refs. [9-25] and references therein. In this work, we focus on the collider searches in the mass range of $3 \mathrm{GeV} \leq m_{\phi} \leq m_{h} / 2$.

In addition to the above generic renormalizable extension, we consider the relaxion framework [26], which offers an alternative approach to the gauge hierarchy problem, and can also provide a DM candidate [27, 28], facilitate baryogenesis [29], and address other hierarchies of the SM [30]. The relaxion is a naturally light pseudo-Nambu-Goldstone field, whose variation in the early Universe induces the scanning of the Higgs mass. The key ingredient of the relaxion mechanism is the so-called backreaction potential, which stops the relaxion evolution at the observed Higgs mass value. The backreaction potential is responsible for the interactions between the Higgs and the relaxion which are relevant for the collider phenomenology, realizing the Higgs portal structure similarly to the generic singlet extension discussed above [19, 20]. The mass range examined in this work, however, represents only the extremely heavy region of the full relaxion parameter space.

The two main parameters in our focus are the Higgs-relaxion mixing and the $h^{2} \phi^{2}$ coupling. We demonstrate that, while having many similarities with the generic portal models, the relaxion is more constrained, but at the same time allows for larger values of the mixing angle than in the generic portal scenarios. This feature occurs because the naturalness considerations, which can be used to constrain the portal parameter space, can be automatically violated by the dynamics of relaxion field [31].

While collider constraints on promptly decaying relaxions or light scalar singlets were derived in ref. [32], here we focus on the range of couplings that makes the scalar sufficiently long-lived such that it does not decay promptly. We take indirect constraints from global Higgs coupling fits as well as direct searches for invisible Higgs decays and displaced and delayed signatures into account. Moreover, we investigate the implications of the bounds on untagged Higgs decays on singlets decaying in the detector. For each method, we evaluate the potential of various hadron and lepton colliders to probe natural parameter space.

The paper is structured as follows. In section 2 we present general properties of the singlet extension. In particular, we consider the renormalizable singlet in section 2.1 and the special case of the relaxion in section 2.2. Section 3 contains the collider bounds ordered by the scalar lifetime. We compare these complementary bounds in section 4 before concluding in section 5 .

\section{Singlet extension of the Higgs sector}

In the following we present the phenomenological features of the real scalar singlet extension of the SM Higgs sector. After discussing the general properties of the scalar, we derive the masses and the relevant couplings for the renormalizable $\mathbb{Z}_{2}$-breaking model, as well as for 
the non-renormalizable relaxion framework. This direct comparison will allow us to make a mapping from one model to the other.

The most general extended scalar potential of the Higgs doublet $H$ and a new singlet scalar $\Phi$ is given by

$$
V_{\mathrm{s}}(\Phi, H)=V(\Phi)+\mu^{2}(\Phi) H^{\dagger} H+\lambda_{h}\left(H^{\dagger} H\right)^{2},
$$

where $V(\Phi)$ and $\mu^{2}(\Phi)$ are functions of the field $\Phi$, whose exact forms depend on the model. We do not consider direct couplings between $\Phi$ and other SM states besides the Higgs. In general, both fields can be written in the unitary gauge as

$$
H=\left(0, \frac{v+h}{\sqrt{2}}\right)^{T}, \quad \Phi=\phi_{0}+\phi
$$

where $v=246 \mathrm{GeV}$ and $\phi_{0}$ are their respective vacuum expectation values (VEVs), and their dynamical degrees of freedom are denoted by $h$ and $\phi$.

If $\Phi$ is not protected by an unbroken $\mathbb{Z}_{2}$ symmetry, the singlet $\phi$ mixes with the Higgs. In this case, $\phi$ inherits the Higgs couplings to the SM particles, suppressed by the mixing angle $\sin \theta$. At the same time, the couplings of the Higgs boson to the SM particles are reduced by a global factor $\cos \theta$. The mass matrix elements are

$$
m_{h h}^{2}=2 v^{2} \lambda_{h}, \quad m_{\phi \phi}^{2}=V^{\prime \prime}\left(\phi_{0}\right)+\frac{1}{2} v^{2} \mu^{2 \prime \prime}\left(\phi_{0}\right), \quad m_{h \phi}^{2}=v \mu^{2 \prime}\left(\phi_{0}\right),
$$

where the derivative is with respect to $\phi$. Defining the mixing angle by

$$
\left(\begin{array}{l}
\phi_{\text {phys. }} \\
h_{\text {phys. }}
\end{array}\right)=\left(\begin{array}{cc}
\cos \theta & -\sin \theta \\
\sin \theta & \cos \theta
\end{array}\right)\left(\begin{array}{l}
\phi \\
h
\end{array}\right),
$$

for $m_{h h}^{2} \gg m_{\phi \phi}^{2}, m_{h \phi}^{2}$, it can be approximated by

$$
\sin \theta \approx \frac{m_{h \phi}^{2}}{m_{h h}^{2}}=\frac{\mu^{2 \prime}\left(\phi_{0}\right)}{2 \lambda_{h} v} .
$$

Since for small mixing angles, the mass and interaction eigenstates are approximately the same, we use the symbols $h$ and $\phi$ for both states throughout the paper and drop the label of the physical mass eigenstates used above. We denote the physical masses by $m_{\phi}$ and $m_{h}$, respectively, and their expressions will be given in the following sections. Due to the mixing, the $\phi$ production from and decay into SM particles equal those of a SM Higgs boson with the respective $\phi$ mass, modified by the mixing angle.

In addition, if the mixing angle or $\mu^{2 \prime \prime}\left(\phi_{0}\right)$ are non-zero, ${ }^{1}$ the Higgs couples to a pair of singlets. We denote this coupling of the mass eigenstates by $c_{h \phi \phi}$, which receives the following contributions

$$
\begin{aligned}
c_{h \phi \phi}= & 3 \mathrm{c}_{\theta} \mathrm{s}_{\theta}^{2} v \lambda_{h}+\left(\frac{1}{2} \mathrm{~s}_{\theta}^{3}-\mathrm{c}_{\theta}^{2} \mathrm{~s}_{\theta}\right) \mu^{2 \prime}\left(\phi_{0}\right)+\left(\frac{1}{2} \mathrm{c}_{\theta}^{3}-\mathrm{c}_{\theta} \mathrm{s}_{\theta}^{2}\right) v \mu^{2 \prime \prime}\left(\phi_{0}\right) \\
& +\frac{1}{4} \mathrm{c}_{\theta}^{2} \mathrm{~s}_{\theta} v^{2} \mu^{2 \prime \prime \prime}\left(\phi_{0}\right)+\frac{1}{2} \mathrm{c}_{\theta}^{2} \mathrm{~s}_{\theta} V^{\prime \prime \prime}\left(\phi_{0}\right),
\end{aligned}
$$

\footnotetext{
${ }^{1}$ For $\phi_{0}=0$ this requires that $\mu^{2}$ contains a $\phi^{2}$ term whereas for $\phi_{0} \neq 0$ higher powers of $\phi$ are also a valid solution. These terms can be explicit or can arise from an expansion in $\phi$.
} 
where we use the shorthand notation $\mathrm{s}_{\theta} \equiv \sin \theta$ and $\mathrm{c}_{\theta} \equiv \cos \theta$. In the two concrete models considered below, this expression simplifies substantially, especially in the limit of small mixing. When $2 m_{\phi}<m_{h}$, the Higgs can decay via the new channel $h \rightarrow \phi \phi$ with a decay width of

$$
\Gamma_{h \rightarrow \phi \phi}=\frac{c_{h \phi \phi}^{2}}{8 \pi m_{h}} \sqrt{1-\frac{4 m_{\phi}^{2}}{m_{h}^{2}}}
$$

\subsection{Renormalizable singlet}

The most general renormalizable form of $V(\Phi)$ and $\mu^{2}(\Phi)$ is

$$
\begin{aligned}
V(\Phi) & =t \Phi+\frac{1}{2} m_{0}^{2} \Phi^{2}+\frac{a_{\phi}}{3} \Phi^{3}+\frac{\lambda_{\phi}}{4} \Phi^{4}, \\
\mu^{2}(\Phi) & =-\mu_{0}^{2}+2 a_{h \phi} \Phi+\hat{\lambda}_{h \phi} \Phi^{2}
\end{aligned}
$$

This theory, often also called a Higgs portal model, has been studied extensively in the literature, see e.g. [22-24, 33-36] and references therein. For later convenience, we choose $\phi_{0}=0$, which can always be obtained by a shift of the $\phi$ field. This implies $t=-a_{h \phi} v^{2}$ from the minimum condition of the scalar potential $V_{\mathrm{s}}$. In this model the mixing angle is

$$
\sin \theta=\frac{1}{\sqrt{2}} \sqrt{1-\frac{1}{\sqrt{1+x_{\mathrm{int}}^{2}}}}=\frac{1}{\sqrt{2}} \sqrt{1-\sqrt{1-x_{\mathrm{phys}}^{2}}} \approx \frac{a_{h \phi}}{v \lambda_{h}}
$$

where $x_{\text {state }}=4 v a_{h \phi} / \Delta m_{\text {state }}^{2}$, with $\Delta m_{\text {int }}^{2}=m_{h h}^{2}-m_{\phi \phi}^{2}$ being the difference of the diagonal entries of the mass matrix before diagonalization, and $\Delta m_{\text {phys }}^{2}=m_{h}^{2}-m_{\phi}^{2}$ being the difference of the physical mass eigenvalues. ${ }^{2}$ The approximation in the last step holds for $\hat{\lambda}_{h \phi} v^{2}+m_{0}^{2} \ll 2 \lambda_{h} v^{2}$ and $a_{h \phi} \ll v \lambda_{h}$. This corresponds to a large mass splitting between the singlet and the Higgs and a small mixing angle. The physical masses are

$$
m_{\phi, h}^{2}=\frac{1}{2}\left(m_{0}^{2}+v^{2}\left(2 \lambda_{h}+\hat{\lambda}_{h \phi}\right) \mp v^{2} \sqrt{\left(\frac{4 a_{h \phi}}{v}\right)^{2}+\left(\frac{m_{0}^{2}}{v^{2}}+\hat{\lambda}_{h \phi}-2 \lambda_{h}\right)^{2}}\right) .
$$

For $\left|a_{h \phi}\right| \ll v \lambda_{h}$ the masses are approximated as

$$
\begin{aligned}
& m_{\phi}^{2} \approx m_{0}^{2}+v^{2} \hat{\lambda}_{h \phi}-\Delta_{m} \\
& m_{h}^{2} \approx 2 v^{2} \lambda_{h}+\Delta_{m} \quad \text { with } \\
& \Delta_{m}=\frac{4 a_{h \phi}^{2}}{2 \lambda_{h}-m_{0}^{2} / v^{2}-\hat{\lambda}_{h \phi}} \approx 2 v^{2} \mathrm{~s}_{\theta}^{2} \lambda_{h} \approx m_{h}^{2} \mathrm{~s}_{\theta}^{2},
\end{aligned}
$$

where the approximations rely on a small mixing and a large splitting of the diagonal entries of the mass matrix, exactly as the approximation in eq. (2.10). Using in eq. (2.6)

\footnotetext{
${ }^{2}$ Hence the difference in the squared physical masses can be expressed as $\Delta m_{\text {phys }}^{2}=\Delta m_{\text {int }}^{2} \sqrt{1+x_{\text {int }}^{2}}$.
} 
the explicit expressions for $V(\Phi)$ and $\mu^{2}(\Phi)$ given in this section, we obtain the explicit coupling $c_{h \phi \phi}$

$$
\begin{aligned}
c_{h \phi \phi} & =3 \mathrm{c}_{\theta} \mathrm{s}_{\theta}^{2} v \lambda_{h}+a_{h \phi}\left(\mathrm{s}_{\theta}^{3}-2 \mathrm{c}_{\theta}^{2} \mathrm{~s}_{\theta}\right)+\hat{\lambda}_{h \phi} v\left(\mathrm{c}_{\theta}^{3}-2 \mathrm{c}_{\theta} \mathrm{s}_{\theta}^{2}\right)+a_{\phi} \mathrm{c}_{\theta}^{2} \mathrm{~s}_{\theta} \\
& \approx \mathrm{s}_{\theta}^{2} v \lambda_{h}+\hat{\lambda}_{h \phi} v+a_{\phi} \mathrm{s}_{\theta} \\
& \approx \mathrm{s}_{\theta}^{2} \frac{m_{h}^{2}}{2 v}+\lambda_{h \phi} v
\end{aligned}
$$

where the approximation holds for small mixing and makes use of eq. (2.10), and we define

$$
\lambda_{h \phi} \equiv \hat{\lambda}_{h \phi}+\mathrm{s}_{\theta} \frac{a_{\phi}}{v} .
$$

We use this as a parameter in the phenomenological investigations.

Theoretical bounds on the parameter space. The relevant phenomenology is described by the four physical parameters $m_{\phi}, s_{\theta}, \hat{\lambda}_{h \phi}$, and $a_{\phi}$. The parameters $\mathrm{s}_{\theta}[33,37]$, $\hat{\lambda}_{h \phi}$, and $a_{\phi}$ contribute to $m_{\phi}$, the former two at tree-level and $a_{\phi}$ via a $\phi$-loop. Therefore, their viable ranges are bounded by naturalness and depend on $m_{\phi}$ as $^{3}$

$$
\begin{aligned}
\sin \theta & \lesssim \frac{m_{\phi}}{m_{h}}, \\
\hat{\lambda}_{h \phi} & \lesssim \frac{m_{\phi}^{2}}{v^{2}}, \\
a_{\phi} & \lesssim 4 \pi m_{\phi} .
\end{aligned}
$$

The upper naturalness bound on $\lambda_{h \phi}$ is then given by

$$
\lambda_{h \phi}^{\max }=\frac{m_{\phi}^{2}}{v^{2}}+4 \pi \frac{m_{\phi}}{v} \mathbf{s}_{\theta} .
$$

As we will see in the specific case of the relaxion, such naturalness bounds may be violated by orders of magnitude as a consequence of the cosmological evolution of the fields.

\subsection{Relaxion}

Unlike the generic Higgs portal model considered above, the relaxion scenario is designed to solve the SM hierarchy problem and is therefore much more constrained and predictive. First, we briefly summarize the cosmological relaxation mechanism [26], considering the relaxion potential of the form

$$
\begin{aligned}
V(\Phi) & =r g \Lambda^{3} \Phi \\
\mu^{2}(\Phi) & =-\Lambda^{2}+g \Lambda \Phi-\tilde{M}^{2} \cos \left(\frac{\Phi}{f}\right) .
\end{aligned}
$$

Here $\Lambda$ is a UV cutoff, $\tilde{M}$ is the height of the backreaction potential (see below) and $f$ is the relaxion oscillation scale. During its evolution, the relaxion scans the Higgs mass parameter $\mu^{2}(\Phi)$ from a large and positive value $\sim \Lambda^{2} \gg v^{2}$ down to negative values.

\footnotetext{
${ }^{3}$ We here neglect the $\log \Lambda$ dependence of the upper limit on $a_{\phi}$.
} 
This scanning is a result of the slow-roll potential $V(\Phi)$, which is controlled by the small dimensionless coupling $g$, and $r>1 / 16 \pi^{2}$ which is bounded from below by the requirement of technical naturalness [38]. Once $\mu^{2}(\Phi)$ becomes negative, the Higgs gets a VEV and thereby activates a backreaction potential $\propto \cos (\Phi / f)$, which eventually stops the rolling of the relaxion at a value $\phi_{0}$, where $v\left(\phi_{0}\right)=246 \mathrm{GeV}$ (see [39] for a recent discussion of the stopping mechanisms).

Such a theory naturally generates a large hierarchy between the electroweak scale and $\Lambda$, solving the SM naturalness problem. ${ }^{4}$ In the following, we require

$$
f \geq \Lambda \geq \Lambda_{\min }=1 \mathrm{TeV}
$$

The backreaction mechanism is model-dependent, and its most general potential is

$$
V_{\mathrm{br}}(h, \phi)=-\tilde{M}^{4-j}\left(\frac{v+h}{\sqrt{2}}\right)^{j} \cos \left(\frac{\phi}{f}\right)
$$

where we chose $j=2$ and assume the minimal scenario of [26] (for alternative scenarios see e.g. refs. [26, 38, 45-48]). To suppress Higgs-independent loop-induced corrections to the backreaction potential [47], the backreaction scale has to satisfy $\tilde{M}^{2} \ll 8 \pi^{2} v^{2}$. Concretely, we require

$$
\tilde{M} \leq \tilde{M}_{\max }=1 \mathrm{TeV}
$$

\subsubsection{Comparison to singlet extension}

Around a local relaxion minimum $\langle\phi\rangle=\phi_{0}$, all the phenomenologically relevant features of the relaxion model can be derived from those of the singlet extension discussed in section 2.1 , by substituting

$$
\begin{aligned}
m_{0}^{2}, a_{\phi}, \lambda_{\phi} & \rightarrow 0, \\
a_{h \phi} & \rightarrow \sin \left(\frac{\phi_{0}}{f}\right) \frac{\tilde{M}^{2}}{2 f}+\frac{g \Lambda}{2}, \\
\hat{\lambda}_{h \phi} & \rightarrow \cos \left(\frac{\phi_{0}}{f}\right) \frac{\tilde{M}^{2}}{2 f^{2}} .
\end{aligned}
$$

Making these substitutions in eqs. (2.10) and (2.12), and omitting the term suppressed by the small coupling $g$, we obtain

$$
\begin{aligned}
\mathrm{s}_{\theta} & \approx \frac{\tilde{M}^{2}}{2 v f \lambda_{h}} \sin \left(\frac{\phi_{0}}{f}\right), \\
m_{\phi}^{2} & \approx \frac{v^{2} \tilde{M}^{2}}{2 f^{2}} \cos \left(\frac{\phi_{0}}{f}\right)-\frac{v^{2}}{m_{h}^{2}} \frac{\tilde{M}^{4}}{f^{2}} \sin ^{2}\left(\frac{\phi_{0}}{f}\right),
\end{aligned}
$$

\footnotetext{
${ }^{4}$ The relaxion does not solve the gauge hierarchy problem up to the Planck scale, and thus requires a UV completion to provide the needed $\Lambda \ll M_{\mathrm{Pl}}$ [40-42], and also to produce a large relaxion field excursion $[43,44]$.
} 
where we neglect small corrections to the Higgs mass $m_{h}$. We notice that all the other couplings can be expressed as functions of $m_{\phi}$ and $\mathrm{s}_{\theta}$ as

$$
\begin{aligned}
\hat{\lambda}_{h \phi}=\lambda_{h \phi} & =\frac{m_{\phi}^{2}}{v^{2}}+\frac{m_{h}^{2}}{v^{2}} \mathrm{~s}_{\theta}^{2}, \\
a_{h \phi} & =\frac{m_{h}^{2}}{2 v} \mathrm{~s}_{\theta} .
\end{aligned}
$$

This means that this relaxion model has only two free parameters relevant for collider phenomenology, i.e. two less than the generic singlet case. The triple scalar coupling $c_{h \phi \phi}$ can then be written as

$$
c_{h \phi \phi} \approx \frac{m_{\phi}^{2}}{v}+\frac{3}{2} \frac{m_{h}^{2}}{v} \mathrm{~s}_{\theta}^{2}
$$

Hence, in contrast to the renormalizable singlet extension that has $\hat{\lambda}_{h \phi}$ and $a_{h \phi}$ as additional parameters, in the relaxion model this coupling is fully determined by $m_{\phi}$ and $\mathrm{s}_{\theta}$. Thus, the viable phenomenological parameter space is more limited and the model is more predictive.

\subsubsection{Theoretical bounds on the parameter space}

Naively, the general naturalness bound on $\mathrm{s}_{\theta}$ obtained in eq. (2.19) applies also to the relaxion model. However, following refs. [28, 31], the dynamical evolution of the relaxion can fix the value of $\phi_{0}$ at such a position that the two contributions to the relaxion mass in eq. (2.32) cancel each other to a high precision, leading to a larger allowed value for $\sin \theta$ for a given mass. In the following, we denote the number of a minimum by $n$.

First minimum. The degree of such a cancellation is maximal in the first local minimum of the relaxion potential. There, in the limit of $\tilde{M} \gg \sqrt{\lambda_{h}} v$, the relaxion mass and mixing angle are given by (see appendix $\mathrm{A})^{5}$

$$
\begin{aligned}
m_{\phi}^{2} & \approx \sqrt{\frac{3 \pi}{2 \lambda_{h}^{1 / 2}} \frac{(v \tilde{M})^{5 / 2}}{f^{2} \Lambda},} \\
\sin \theta & \approx \frac{\tilde{M}}{2 \sqrt{\lambda}_{h} f} .
\end{aligned}
$$

The mixing is maximized for maximal $\tilde{M}$ and minimal $f$, namely $f=\Lambda$. Expressing $f$ in terms of $m_{\phi}$ from eq. (2.36) and substituting this in eq. (2.37) yields

$$
\sin \theta<\left(\frac{\tilde{M}_{\max }}{96 \pi v \lambda_{h}^{5 / 2}}\right)^{1 / 6}\left(\frac{m_{\phi}}{v}\right)^{2 / 3} .
$$

Thus, the mixing is parametrically enhanced, as it is proportional to $\left(m_{\phi} / v\right)^{2 / 3}$ instead of the naturally expected $\propto m_{\phi} / v$, with the prefactor of $\mathcal{O}(1)$. A relaxion with a larger mixing than that defined in eq. (2.38) corresponds to an unnatural tuning of the relaxion mass.

\footnotetext{
${ }^{5}$ Obtaining $m_{\phi}$ in the $\mathrm{GeV}$ range necessitates a large value of $\tilde{M}$, and therefore the limit $\tilde{M} \gg \sqrt{\lambda_{h}} v$ is justified, see eq. (2.32).
} 
Solving eq. (2.36) for $f$, substituting it in eq. (2.37), and setting $\Lambda(\tilde{M})$ to its minimal (maximal) value, we obtain the lower bound on the mixing angle

$$
\sin \theta \simeq\left(\frac{1}{24 \pi \lambda_{h}^{3 / 2}}\right)^{1 / 4} \frac{m_{\phi} \Lambda^{1 / 2}}{v^{5 / 4} \tilde{M}^{1 / 4}}>\left(\frac{\Lambda_{\min }^{2}}{24 \pi \lambda_{h}^{3 / 2} v \tilde{M}_{\max }}\right)^{1 / 4} \frac{m_{\phi}}{v} \approx \frac{m_{\phi}}{v} .
$$

Generic minimum. As mentioned above, the degree of tuning decreases if the relaxion stops in a later minimum. This may happen either through quantum fluctuations or by classical rolling $[49,50]$. In the limit of small tuning in a far minimum, $n \gg 1, \sin \left(\phi_{0} / f\right) \sim$ $\cos \left(\phi_{0} / f\right) \sim \mathcal{O}(1)$ and naturalness arguments lead to an estimate of the minimal value of the mixing angle. In this limit, the mass can be approximated as

$$
m_{\phi}^{2} \simeq \frac{v^{2} \tilde{M}^{2}}{2 f^{2}}
$$

while the mixing angle reads

$$
\sin \theta \simeq \frac{\tilde{M}^{2}}{2 \lambda_{h} f v}
$$

Expressing $\tilde{M}$ through the relaxion mass, and using the lower bound on $f$ leads to a lower bound on the mixing,

$$
\sin \theta \simeq \frac{m_{\phi}^{2} f}{\lambda_{h} v^{3}}>\frac{m_{\phi}^{2} \Lambda_{\min }}{\lambda_{h} v^{3}} .
$$

For the relaxion in such a minimum, and also for generic untuned Higgs portal models, the maximal mixing is given by eq. (2.19). All the $\sin \theta$ bounds derived in this section are valid up to order-one factors and thus should not be taken as exact.

Combined constraints: the relaxion band. As follows from the above discussion, for each mass $m_{\phi}$ there is a relaxion-specific lower and upper bound on $\sin \theta$. The upper bound arises from the first minimum, see eq. (2.38), and always exceeds the upper bound for the relaxion in a generic minimum. For $f_{\min }=\Lambda_{\min }=\tilde{M}_{\max }=1 \mathrm{TeV}$, the overall lower bound stems from the general minimum for $m_{\phi} \leq 8 \mathrm{GeV}$ and from the first minimum otherwise. This crossover causes a kink of the lower bound. The range of natural values of $\sin \theta$ for a given mass will appear as the relaxion band in the plots in the phenomenological analyses.

Figure 1 shows the lines in the $\sin ^{2} \theta-\lambda_{h \phi}$ plane which fulfill the relaxion relation for $\lambda_{h \phi}$ as a function of $m_{\phi}$ and $\sin \theta$ within the $\sin ^{2} \theta$ range bounded by naturalness of the first and generic minima. The dashed part of the lines corresponds to $\sin ^{2} \theta<m_{\phi}^{2} / v^{2}$, i.e. the naturalness limit of the renormalizable singlet. The solid line segments represent $\sin ^{2} \theta>m_{\phi}^{2} / v^{2}$, i.e. values that are unnatural for the renormalizable singlet, but still natural for the relaxion.

\section{Collider bounds on (long-lived) scalar singlets}

We present bounds on scalar singlets for a broad range of their lifetime. This necessitates a combination of various search strategies. Central to them is the lifetime which is shown 


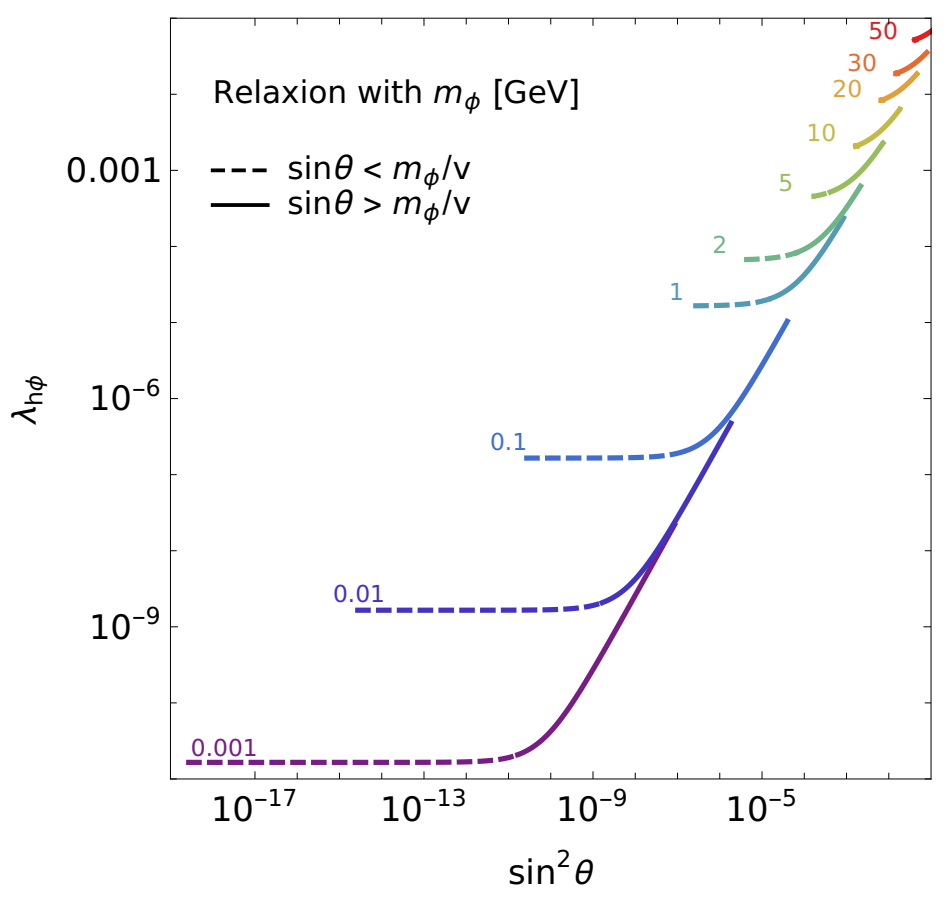

Figure 1. Natural relaxion parameter space in the $\sin ^{2} \theta-\lambda_{h \phi}$ plane. Each color shows one mass given in $\mathrm{GeV}$. The dashed (solid) part of the lines corresponds to $\sin ^{2} \theta<(>) m_{\phi}^{2} / v^{2}$, i.e. where the mixing angle of the renormalizable singlet is natural (unnatural). The plotted $\lambda_{h \phi}\left(m_{\phi}, \sin \theta\right)$ of the relaxion is defined in eq. (2.33), within the natural $\sin ^{2} \theta$ range from section 2.2.2.

in figure 2 for the relevant masses and mixing angles. For

- short lifetimes, untagged Higgs decays into a pair of singlets lead to strong indirect bounds;

- intermediate lifetimes, displaced vertex (DV) searches and strategies based on timing information probe a broad range of the parameter space;

- long lifetimes, the singlet escapes the detector and can account for invisible signatures.

We compare these bounds to the ones previously studied from direct searches in $Z$ decays and from associated $Z \phi$ production. The presented bounds are based on singlet pair production via Higgs decays $(h \rightarrow \phi \phi)$. The production via singlet-Higgs mixing is negligible for the parameter region considered here, for details see appendix B. Our bounds apply to the general singlet extension of section 2.1. We will point out which regions of the displayed parameter space can also be realized by the relaxion.

\subsection{Fits of untagged and invisible Higgs decays}

Beyond the Standard Model (BSM) physics can modify the tagged Higgs branching ratios both by modifying the Higgs couplings to SM particles by $\kappa_{x}=c_{x} / c_{x}^{\mathrm{SM}}$, and by introducing new decay channels for the Higgs, depleting the relative SM contribution to the total decay 


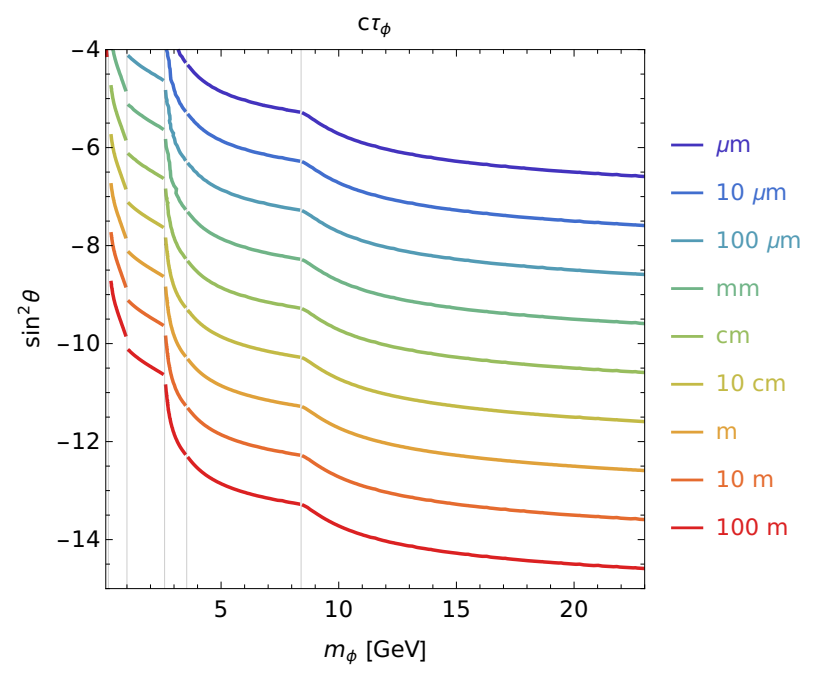

Figure 2. Decay length of the singlet [19] depending on its mass $m_{\phi}$ and mixing angle $\sin \theta$.

width $[32,51]$

$$
\mathrm{BR}_{h \rightarrow x}=\frac{\kappa_{x}^{2} \Gamma_{h \rightarrow x}^{\mathrm{SM}}}{\sum_{y \in \mathrm{SM}} \kappa_{y}^{2} \Gamma_{y}^{\mathrm{SM}}+\Gamma_{\mathrm{BSM}}} \approx \mathrm{BR}_{h \rightarrow x}^{\mathrm{SM}}\left(1-\mathrm{BR}_{\mathrm{BSM}}\right) .
$$

The BSM particles produced in these Higgs decays can either decay visibly, or remain invisible. While searches for Higgs decays with missing energy directly constrain the invisible branching $\mathrm{BR}_{\text {inv }}$, these search results can also be used as a tagged category in a fit. In contrast, the final states of the visible BSM Higgs decays (e.g. light jets) are generally not included in the list of tagged visible decays ( such as $h \rightarrow \tau \tau, b b, V V, \ldots$, explicitly displayed e.g. in table 1 of ref. [52]). Hence they remain untagged, ${ }^{6}$ and the corresponding Higgs branching $\mathrm{BR}_{\text {unt }}$ is not determined by any specific search, but by the uncertainties of the tagged channels. Therefore, global fits of the Higgs coupling modifiers $\kappa_{x}$ to measured signal strengths $\mu_{i f}=\sigma_{i} / \sigma_{i}^{\mathrm{SM}} \cdot \mathrm{BR}_{f} / \mathrm{BR}_{f}^{\mathrm{SM}}$ (tagged production cross sections times tagged branching ratios normalized to the SM prediction), together with searches for invisible Higgs decays, allow to constrain the Higgs decay width into BSM particles, ${ }^{7}$ $\Gamma_{\mathrm{BSM}}=\Gamma_{\mathrm{inv}}+\Gamma_{\mathrm{unt}}$.

The global Higgs fits performed in the scope of the European Strategy Update [56] present results for the future hadron colliders HL-LHC, LHeC, HE-LHC and FCChh, as well as for the lepton colliders ILC, CLIC, CEPC and FCCee running at different energy stages. Here we apply the results from the so-called kappa-2 scenario that treats $\mathrm{BR}_{\text {inv }}$ and $\mathrm{BR}_{\mathrm{unt}}$ as free parameters for each collider individually. In addition, it has several independent $\kappa_{x}$ whereas in the general singlet and the relaxion models there is only one overall $\kappa \equiv \cos \theta$, see also ref. [32]. Furthermore, in the region of intermediate and high $\sin \theta \gtrsim 10^{-11}$, such that $c \tau_{\phi}\left(m_{\phi}, \sin \theta\right) \lesssim 1 \mathrm{~m}$ for $m_{\phi} \geq 5 \mathrm{GeV}$ (see figure 2 ), all $\phi$ s decay

\footnotetext{
${ }^{6}$ For the implications of the direct searches for $h \rightarrow \phi \phi$ further decaying promptly into four visible particles, e.g. $h \rightarrow 4 b, h \rightarrow b b \tau \tau$, see refs. [32, 53-55].

${ }^{7}$ The SM contributions to $\Gamma_{\text {inv }}$ and $\Gamma_{\text {unt }}$ are subtracted.
} 
inside the detector, hence $\mathrm{BR}_{\text {inv }}=0$, and fitting only two parameters, $\kappa$ and $\mathrm{BR}_{\text {unt }}$, would be sufficient. In the opposite case of very small $\sin \theta$, the Higgs couplings to SM particles become SM-like $(\kappa \simeq 1)$, and fitting only $\mathrm{BR}_{\text {inv }}$ would be enough. Hence, the multi-parameter fits used in table 1 and in figure 3 give rise to conservative bounds on this actually more predictive model, defined by less parameters. To evaluate the gain in sensitivity by fitting only the needed parameters, we also include the dedicated fit results performed for the CLIC stages [53], see the lower part of table 1.

A combination of the ATLAS and CMS data collected in Run-1 leads to a limit on $\mathrm{BR}_{\mathrm{BSM}}<20 \%$ [51] (which can be applied as a conservative bound on $\mathrm{BR}_{\text {unt }}$ ), comparable to that of ATLAS alone in Run-2 of $\mathrm{BR}_{\text {unt }}<21 \%$ [57]. The strong result of the Run-1 combination, despite the smaller summed luminosity, is due to the fit of only a global $\kappa$ and $\mathrm{BR}_{\mathrm{BSM}}$. A Run-2 combination or a dedicated 2-parameter fit will be able to exclude further parameter space based on the already existing data.

In figure 3 we show the constraints on the $m_{\phi}-\sin ^{2} \theta$ parameter plane of the relaxion. In addition, we show in gray the natural relaxion band, whose upper and lower $\sin \theta$ limits are discussed in section 2.2. The experimental limits and projections result from requiring

$$
\mathrm{BR}_{h \rightarrow \phi \phi}\left(m_{\phi}, \sin ^{2} \theta\right)=\frac{\Gamma_{h \rightarrow \phi \phi}}{\left(1-\sin ^{2} \theta\right) \Gamma_{\text {tot }}^{\mathrm{SM}}+\Gamma_{h \rightarrow \phi \phi}} \leq \mathrm{BR}_{\text {unt }}
$$

where the partial width $\Gamma_{h \rightarrow \phi \phi} \propto c_{h \phi \phi}^{2}$ is given in eq. (2.35), and the total Higgs width in the $\mathrm{SM}$ is $\Gamma_{\text {tot }}^{\mathrm{SM}}=4.1 \mathrm{MeV}[58]$. The contours form horizontal and vertical asymptotes determined by the $\sin ^{2} \theta$ and $m_{\phi}$ contributions to $c_{h \phi \phi}$, respectively. When neglecting the kinematical mass dependence of $\Gamma_{h \rightarrow \phi \phi}$ (for $m_{\phi} \ll m_{h} / 2$ ) and the BSM contribution to the total width, the location of the asymptotes for the relaxion can be approximated as

$$
\begin{aligned}
\left.\sin ^{2} \theta\right|_{m_{\phi} \rightarrow 0} & \approx \frac{4 v}{3} \sqrt{\frac{2 \pi \mathrm{BR}_{\mathrm{unt}} \Gamma_{\mathrm{tot}}^{\mathrm{SM}}}{m_{h}^{3}}} \simeq 0.038 \sqrt{\mathrm{BR}_{\mathrm{unt}}} \\
\left.m_{\phi}\right|_{\sin ^{2} \theta \rightarrow 0} & \approx\left(8 \pi v^{2} m_{h} \Gamma_{\mathrm{tot}}^{\mathrm{SM}} \mathrm{BR}_{\mathrm{unt}}\right)^{1 / 4} \simeq 30 \mathrm{BR}_{\mathrm{unt}}^{1 / 4} .
\end{aligned}
$$

The shaded blue area is already ruled out by Run-1 of the LHC, excluding natural mixing angles of heavy relaxions above $m_{\phi} \gtrsim 18 \mathrm{GeV}$. As indicated in table 1 , this Run-1 bound is in fact on $\mathrm{BR}_{\mathrm{BSM}}$, whereas for such large values of $\sin ^{2} \theta$ all relaxions decay inside the detector. Hence, a specific bound on $\mathrm{BR}_{\text {unt }}$ will exclude also lighter relaxions. The strongest bound will be reached by the FCChh, excluding $m_{\phi} \gtrsim 10 \mathrm{GeV}$ and $\sin ^{2} \theta \gtrsim 3 \cdot 10^{-3}$. As indicated by the dash-dotted yellow lines, the fit of only $\mathrm{BR}_{\mathrm{BSM}}$, assuming all $\kappa_{x}=1$, for CLIC leads to a significant improvement of the bound compared to the multi- $\kappa$ fit at CLIC. ${ }^{8}$ Dedicated fits for the FCCee and FCChh could have the potential to close the high-mass relaxion window above few $\mathrm{GeV}$.

\footnotetext{
${ }^{8}$ Strictly, this fit is applicable only for vanishing $\sin ^{2} \theta$, but in any case the exclusion contour of CLIC380 (CLIC3000) reaches only $\sin ^{2} \theta \simeq 3 \cdot 10^{-3}\left(1.5 \cdot 10^{-3}\right)$ corresponding $\delta \kappa \equiv 1-\cos \theta \simeq 1.5 \cdot 10^{-3}\left(7.6 \cdot 10^{-4}\right)$, i.e. just below the resolution of $\kappa$, see table 1 . Hence we use this fit as an illustration of the gain of sensitivity in a suitable fit of such a predictive model.
} 


\begin{tabular}{|l|c|c|c|c|l|}
\hline Collider & $\sqrt{s}[\mathrm{TeV}]$ & $\mathcal{L}_{\text {int }}\left[\mathrm{ab}^{-1}\right]$ & $\mathrm{BR}_{\text {unt }}[\%]$ & $\delta \kappa[\%]$ & Ref. \\
\hline LHC1 & 7,8 & 0.022 & $20 \diamond$ & 26 & {$[51]$ table 8,1} \\
LHC3 $(\mathrm{S} 2)$ & 13 & 0.3 & $12.3 \triangle$ & 8.6 & {$[51]$ table 11} \\
\hline HL-LHC & 14 & 6 & 4 & 0.99 & \\
HE-LHC (S2) & 27 & 15 & 3.2 & 0.99 & {$[56]$ table 28} \\
LHeC & 1.3 & 1 & 2.2 & 0.99 & \\
\hline ILC250 & 0.25 & 2 & 1.8 & 0.3 & [56] table 29 \\
ILC500 & $0.25,0.35,0.5$ & $2+0.2+4$ & 1.4 & 0.24 & \\
ILC1000 & $0.25,0.35,0.5,1$ & $2+0.2+4+8$ & 1.3 & 0.24 & \\
\hline CEPC & $M_{Z}, 2 M_{W}, 0.24$ & $16+2.6+5.6$ & 1.1 & 0.19 & {$[56]$ table 29} \\
\hline FCCee240 & 0.24 & 5 & 1.2 & 0.21 & \\
FCCee365 & 0.365 & 1.7 & 1.1 & 0.18 & {$[56]$ table 29} \\
FCCee/eh/hh & 100 & 30 & 1 & 0.17 & \\
\hline TeraZ & $M_{Z}$ & $N_{Z}=10^{12}$ & & & \\
\hline CLIC380 & 0.38 & 1 & 2.7 & 0.5 & \\
CLIC1500 & 1.5 & 2.5 & 2.4 & 0.39 & {$[56]$ table 29} \\
CLIC3000 & 3 & 5 & 2.4 & 0.38 & \\
\hline CLIC380 & 0.38 & 1 & $0.92 \star$ & $0.58 \diamond$ & \\
CLIC1500 & 1.5 & 2.5 & $0.39 \star$ & $0.57 \diamond$ & {$[53]$ table 6 } \\
CLIC3000 & 3 & 5 & $0.26 \star$ & $0.57 \diamond$ & \\
\hline
\end{tabular}

Table 1. Upper bounds on $\operatorname{BR}(h \rightarrow$ unt $)$ at $95 \%$ CL from global fits of Higgs signal strengths for different colliders. $\diamond$ : 2-parameter fit of $\kappa$ and $\mathrm{BR}_{\mathrm{BSM}} ; \triangle$ : fit of multiple $\kappa_{x}$ and $\mathrm{BR}_{\mathrm{BSM}} ; \star$ : 1-parameter fit of $\mathrm{BR}_{\mathrm{BSM}}$ (applicable to low $\sin \theta$ because $\kappa \equiv 1$ ); if not labeled, then multi- $\kappa$ fit of $\mathrm{BR}_{\mathrm{unt}}$. $\mathrm{BR}_{\mathrm{BSM}}$ can be interpreted as a conservative $\mathrm{BR}_{\mathrm{unt}}$ bound. The LHC Run-3 bound at approximately $95 \%$ CL was obtained by multiplying the $68 \%$ CL bound by 1.3 , the ratio of the quantiles of a $\chi^{2}$ distribution with 7 parameters. $\delta \kappa$ denotes the $68 \%$ CL uncertainty of the modifier of the most precisely determined Higgs coupling, i.e. $\delta \kappa_{Z}$ (except for the high-energy stages of CLIC where $\delta \kappa_{W}$ is smaller).

The situation is different for the general singlet model where $\lambda_{h \phi}$ is a free parameter and $\mathrm{BR}_{h \rightarrow \phi \phi}$ varies with the choice of $\lambda_{h \phi}$. For a larger value of $\lambda_{h \phi}$ than the one predicted within the relaxion framework, the bounds from untagged Higgs decays can become even stronger, whereas they get reduced to the $\sin ^{2} \theta$ dependence in eq. (2.17) if $\lambda_{h \phi}$ is suppressed. For a fixed $\lambda_{h \phi}$, the bounds only depend on $\sin ^{2} \theta$ (up to the kinematical mass dependence), however, for small enough masses, any fixed value of $\lambda_{h \phi}$ will eventually become unnatural, see eq. (2.22). The naturalness upper bound on the mixing angle for the singlet is shown as the dashed blue line (within the relaxion band).

In general, while the bounds on $\mathrm{BR}_{\mathrm{BSM}}$ hold for arbitrary values of $\sin \theta$, the more specific bounds on $\mathrm{BR}_{\text {unt }}$ are valid as long as the decay length is significantly smaller than the detector size. Conversely, the bounds on $\mathrm{BR}_{\text {inv }}$ apply to decay lengths clearly exceeding the detector size. 


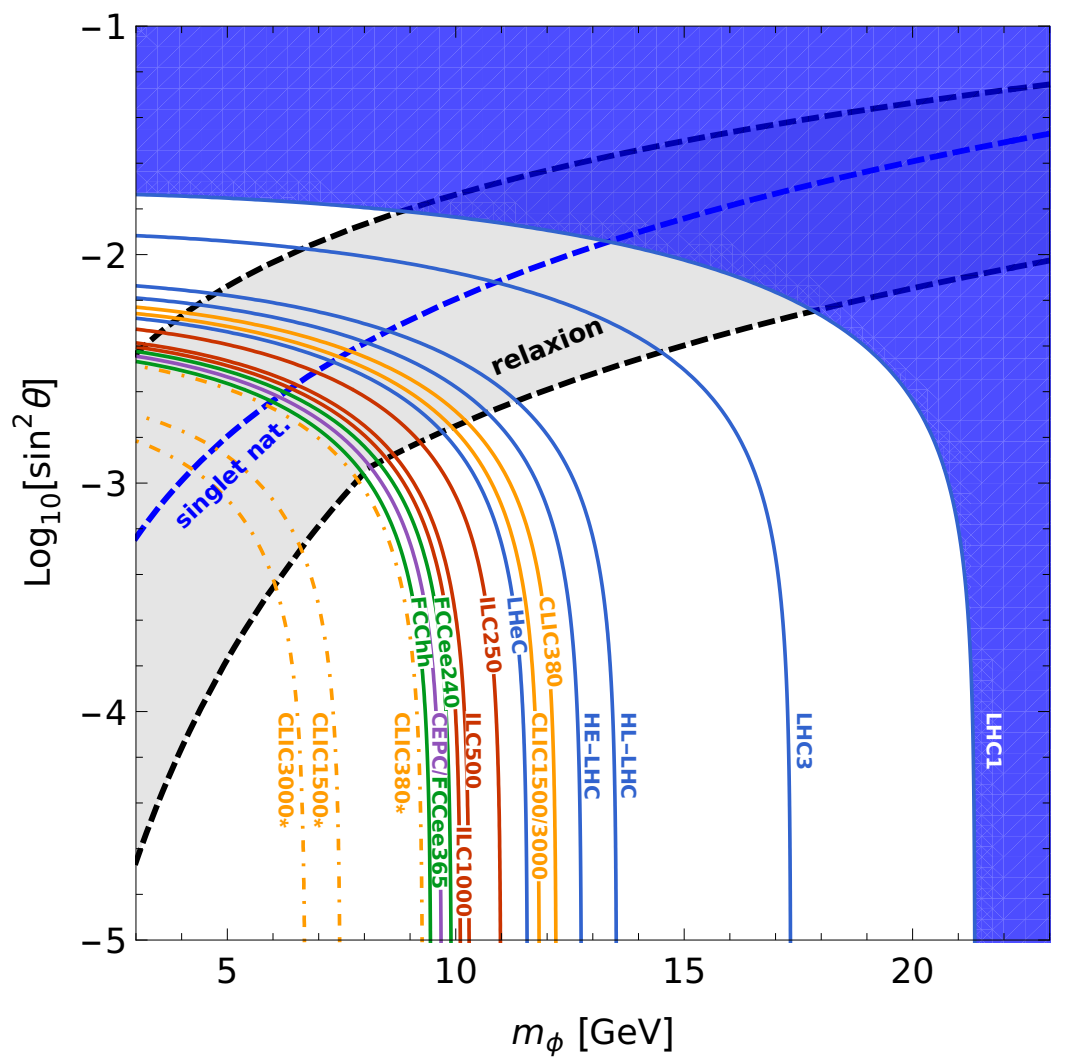

Figure 3. Existing and projected constraints on $\sin ^{2} \theta$ and $m_{\phi}$ from bounds on the branching ratio of Higgs to untagged or BSM final states listed in table 1. The blue shaded area is already excluded. The limits from FCCee at $365 \mathrm{GeV}$ and CEPC coincide (purple). CLIC at $3 \mathrm{TeV}$ does not improve the CLIC limit at $1.5 \mathrm{TeV}$ (solid yellow). The dash-dotted bounds for CLIC labelled by a $*$ indicate the sensitivity from the 1 -parameter fit to $\mathrm{BR}_{\mathrm{BSM}}$ valid in the limit $\sin ^{2} \theta \ll 1$. The dashed dark blue line represents the upper naturalness bound $\sin \theta \leq m_{\phi} / m_{h}$ on the singlet from eq. (2.19). The gray band within the black dashed lines is the natural relaxion range defined by eqs. (2.38), (2.39) and (2.42).

\subsection{Displaced jets}

The singlet can be detected in searches for Higgs decays into displaced jets if it is sufficiently long-lived, but still decays in the detector. ATLAS searches [59-61] and FCC-ee projections [62] provide upper bounds on the branching ratio $\mathrm{BR}_{h \rightarrow \phi \phi}$ as a function of the proper decay length $c \tau_{\phi}$ for a few singlet masses. ${ }^{9}$ We transform them into upper limits on $\lambda_{h \phi}$ as a function of $\sin ^{2} \theta$, for the corresponding mass points given in the analyses, shown in figure 4 . The dashed lines show the upper limit on $\lambda_{h \phi}$ from naturalness, see eq. (2.22). While for $m_{\phi}=5 \mathrm{GeV}$ the ATLAS searches do not constrain any natural parameters of the singlet model, for higher masses the searches already probe parts of the natural parameter region. In contrast, FCC-ee will access natural parameter space for all masses. The displayed FCC-ee bounds show the combination of the two analysis strategies

\footnotetext{
${ }^{9}$ For Higgs decays into complex singlets in searches for displaced jets at the LHeC, see the recent ref. [63].
} 


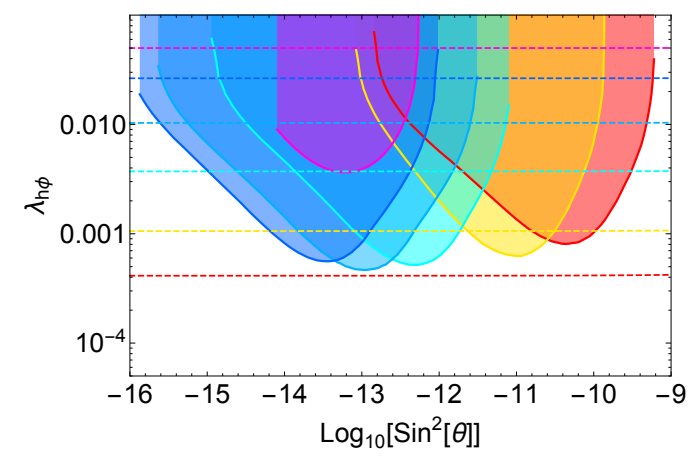

(a) ATLAS $\sqrt{s}=13 \mathrm{TeV}$ with $36 \mathrm{fb}^{-1}$.

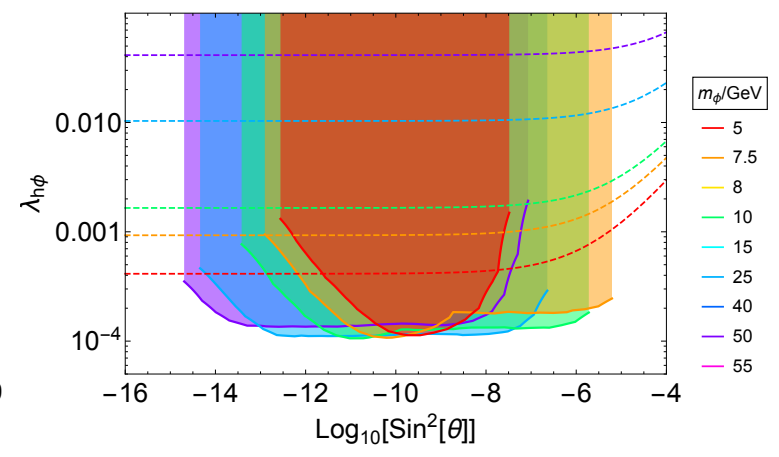

(b) FCC-ee $\sqrt{s}=240 \mathrm{GeV}$ with $5 \mathrm{ab}^{-1}$.

Figure 4. Bounds on $\lambda_{h \phi}$ and $\sin ^{2} \theta$ for various singlet masses arising from searches for displaced jets in Higgs decays. The dashed lines show the upper naturalness limit $\lambda_{h \phi}^{\max }=m_{\phi}^{2} / v^{2}+4 \pi m_{\phi} \mathrm{s}_{\theta} / v$.

from ref. [62], and therefore span a larger range of $\sin ^{2} \theta$. The CLIC sensitivity to a longlived scalar singlet via displaced vertex searches was studied in ref. [64] and is included in our overview plot in figure 9. The comparison shows that CLIC and FCCee provide a comparable sensitivity.

\subsection{Delayed jets}

A powerful strategy to search for long-lived particles was recently presented in ref. [65], allowing to detect displaced vertices in the CMS tracker. ${ }^{10}$ This proposal utilizes the timing detector layer, to be installed at the high luminosity (HL)-Large Hadron Collider (LHC) [69], to identify secondary vertices by the delayed arrival, $\Delta t$, of the light decay products, compared to the arrival time expected for a directly travelling SM particle. An initial state radiation (ISR) jet is used to time-stamp the collision. Ref. [65] provides the bounds for the benchmark scalar masses of $m_{\phi}=10 \mathrm{GeV}$ and $50 \mathrm{GeV}$ at the HLLHC. In order to determine the mass dependence of the experimental reach, we simulate Higgs events at the LHC and FCC-ee, using MadGraph5 [70] at leading order (LO), where the Higgs decays by $h \rightarrow \phi \phi$, and each scalar decays through $\phi \rightarrow j j$. Subsequently, we implement the search strategy presented in [65], reproduce its results, and apply it to the additional mass points. For the FCC-ee, we assume a (hypothetical) timing detector comparable to the one planned for the HL-LHC. The detection efficiency is mostly affected by demanding a long time delay of the jet produced in the singlet decay, related to the singlet's path through the detector, along with requiring the singlet to decay between the inner tracker and the timing layer. Hence, the selection criteria for this search are mainly geometrical. Therefore, for each event kinematics and for each jet $j$ in the event, we find the range of lab frame singlet decay lengths $l_{\phi}$ for which an event will be accepted. Since

\footnotetext{
${ }^{10}$ The new proposal in ref. [66] evaluates the sensitivity of the High Granularity Calorimeter of the CMS detector upgrade to the same type of $h \rightarrow \phi \phi$ decays. While the conservative estimate yields bounds comparable to the timing bounds of ref. [65], only the analysis assuming a displaced track trigger could improve them. This highlights the importance of implementing a such a trigger as proposed in e.g. refs. [67, 68].
} 


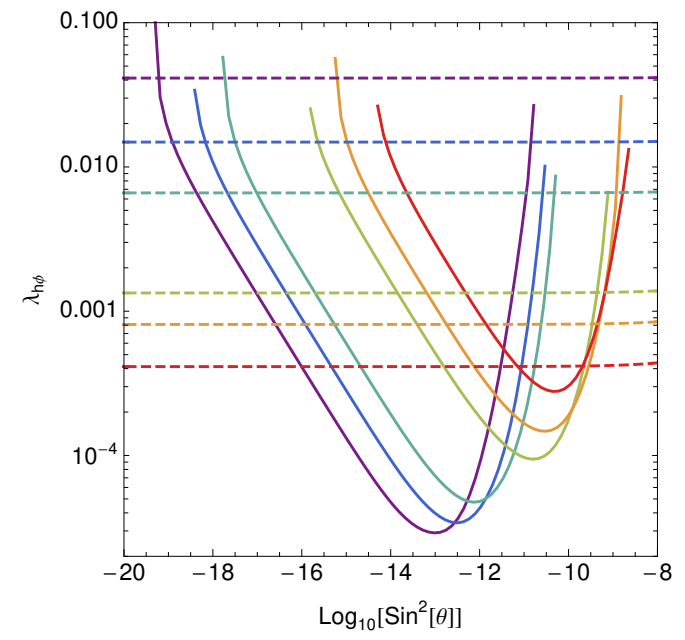

(a) HL-LHC, $\sqrt{s}=14 \mathrm{TeV}$.

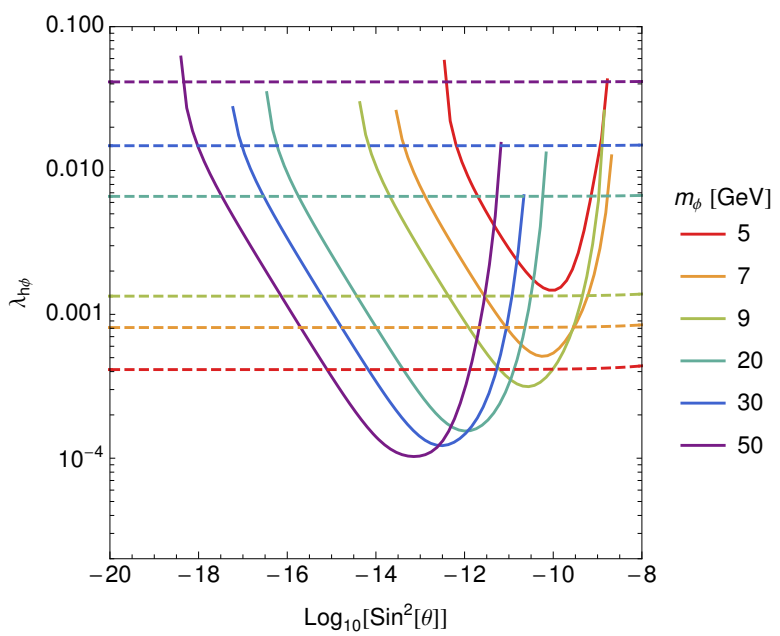

(b) FCC-ee, $\sqrt{s}=240 \mathrm{GeV}$.

Figure 5. Bounds on $\lambda_{h \phi}$ and $\sin ^{2} \theta$ for various singlet masses arising from searches for delayed jets in Higgs decays. The dashed lines show the upper naturalness limit $\lambda_{h \phi}^{\max }$ for each mass.

the detection of a single delayed jet is sufficient, each event is then weighed by the event efficiency $\epsilon_{\text {event }}=1-\left(1-w_{1}\right)\left(1-w_{2}\right)\left(1-w_{3}\right)\left(1-w_{4}\right)$, where $w_{j}$ is the probability of $\phi$ to decay within the allowed region, which is calculated from an exponential distribution

$$
w_{j}=\frac{1}{c \tau_{\phi} \gamma_{\phi} \beta_{\phi}} \int_{l_{\phi, j} \text { allowed }} \exp \left(-\frac{l}{c \tau_{\phi} \gamma_{\phi} \beta_{\phi}}\right) \mathrm{d} l .
$$

More details on the calculation, as well as on the resulting efficiencies and the expected upper limits on $\mathrm{BR}_{h \rightarrow \phi \phi}$ as a function of $c \tau_{\phi}$ can be found in appendix $\mathrm{C}$.

The interpretation of these bounds in terms of the singlet parameters $\lambda_{h \phi}$ and $\sin ^{2} \theta$ is shown in figure 5 . While the HL-LHC probes natural values of $\lambda_{h \phi}$ for $m_{\phi}>5 \mathrm{GeV}$, at the FCC-ee this is the case only for slightly higher masses. As this analysis has almost zero background in the signal region of $\Delta t>1 \mathrm{~ns}$ (for details see ref. [65]), its sensitivity is determined by the number of Higgses. Therefore, the HL-LHC appears to perform better than the FCCee. Since it is the hadronic environment at the HL-LHC that necessitates this restrictive cut on $\Delta t$, the FCCee can allow for a looser cut, and the limit presented here based on the HL-LHC cut is conservative.

\subsection{Searches for invisible Higgs decays}

If the proper decay length of the scalar is larger than, or comparable to, the size of the detector, the scalar may give rise to missing energy. Global Higgs coupling fits set strong bounds on $\mathrm{BR}_{h \rightarrow \text { inv }}[56]$. These can be interpreted as bounds on $\lambda_{h \phi}$ in the limit of vanishing $\sin \theta$, i.e. infinite lifetime. To investigate the region of intermediate lifetimes where only a fraction of the scalars escape the detector, we need to make use of direct searches for invisible Higgs decays. To take this fraction into account, we recast the analysis by CMS and the studies for the HL-LHC and FCCee listed in table 2 to constrain the appropriate 


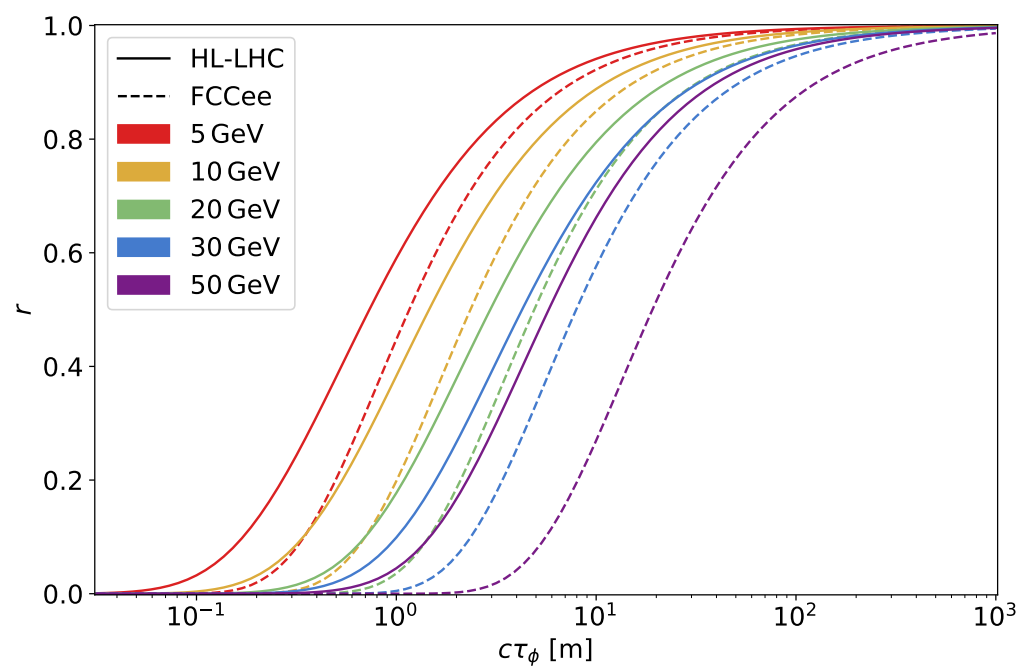

Figure 6. The rescaling factor $r$ defined in eq. (3.6) as a function of $c \tau_{\phi}$ for the HL-LHC and FCCee. The dependence at the LHC is comparable to the HL-LHC. The larger $r$, the more singlets escape the detector before decaying.

region of the singlet parameter space. The bounds given by these searches need to be weakened by a factor $r$, accounting for the cases where both scalars decay outside the detector. The rescaling factor $r$ is obtained by

$$
r=\frac{1}{N} \sum_{i=1}^{N} \exp \left(-\frac{m_{\phi}}{c \tau_{\phi}}\left(\frac{L_{i_{1}}}{p_{i_{1}}}+\frac{L_{i_{2}}}{p_{i_{2}}}\right)\right),
$$

where the sum runs over all $h \rightarrow \phi \phi$ events passing the selection criteria when an infinite decay length is assumed, $p$ is the momentum of each scalar, $L$ is the distance the scalar travels inside the detector, and the indices $\{1,2\}$ mark the two scalars produced in the Higgs decay. A conservative estimate of the rescaled bound can be given by minimizing $r$ for each search. For LHC searches, which require a large missing $p_{T}$, this can be approximated by $r_{\mathrm{LHC}}^{\text {consv. }} \approx \exp \left(-\frac{4 L_{T} m_{\phi}}{c \tau_{\phi} p_{T}^{\text {miss }}}\right)$ where $L_{T}$ is the transverse detector size and $p_{T}^{\text {miss }}$ is the minimally required missing transverse momentum. For lepton colliders, such as the FCC-ee with a lower $\sqrt{s}=240 \mathrm{GeV}$, a better approximation is given by setting the energy of each scalar to $m_{h} / 2$, as the Higgs is produced at low momentum, i.e. $r_{\text {FCC-ee }}^{\text {consv. }} \approx \exp \left(-\frac{4 L m_{\phi}}{c \tau_{\phi} \sqrt{m_{h}^{2}-4 m_{\phi}^{2}}}\right)$.

For a more precise estimate of the bounds, we determine $r$ for each search in table 2 . We use MadGraph5 [70] to simulate the leading signal process in each search at LO. We then apply their selection cuts, and obtain the $\left(\frac{L_{i_{1}}}{p_{i_{1}}}+\frac{L_{i_{2}}}{p_{i_{2}}}\right)$ distribution for each signal mass, and subsequently obtain $r$ following eq. (3.6). The signal processes and selection cuts applied are summarized in table 3. The resulting $r$ for the HL-LHC and FCC-ee is shown in figure 6 as a function of $c \tau_{\phi}$. For a given $m_{\phi}$ and $c \tau_{\phi}, r$ is larger for the HL-LHC because the $L / p$ distributions peak at lower values than for the FCC-ee.

The CMS bounds as well as the HL-LHC and FCCee projections on $\lambda_{h \phi}$ and $\sin ^{2} \theta$ are shown for different values of $m_{\phi}$ in figure 7 . In general, each contour has a horizontal and 


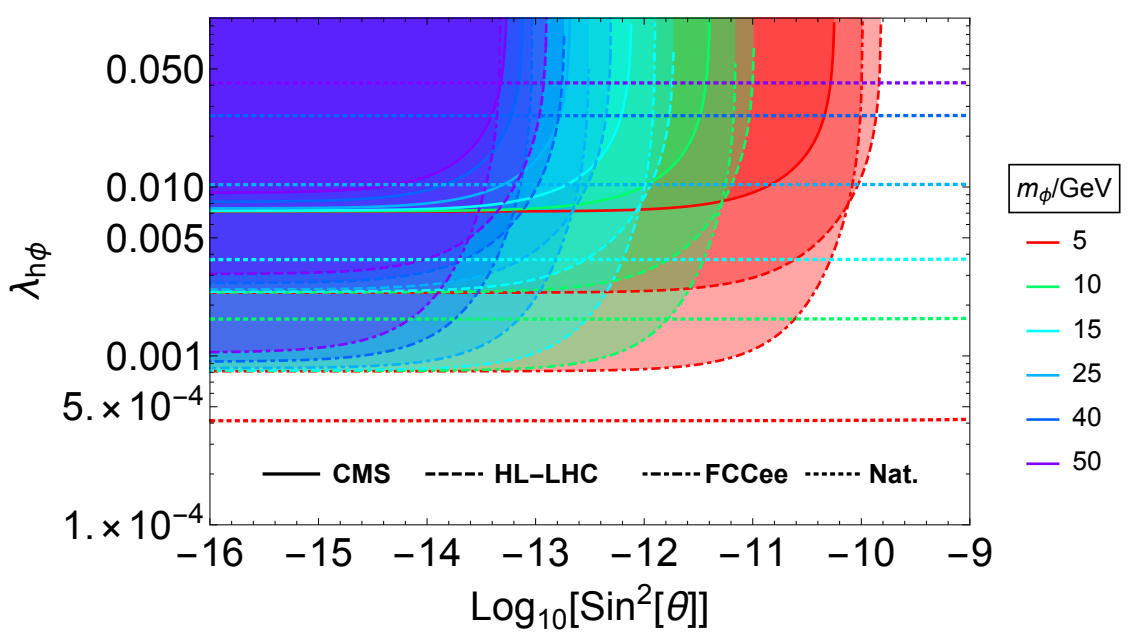

Figure 7. Bounds on $\lambda_{h \phi}$ and $\sin ^{2} \theta$ for various singlet masses arising from searches for invisible Higgs decays. The dotted lines show the upper naturalness limit $\lambda_{h \phi}^{\max }$ for each mass.

a vertical asymptote, driven by the limit on $\mathrm{BR}_{h \rightarrow \phi \phi}$ and by the lifetime, respectively. As a consequence, the horizontal asymptotes are hardly mass dependent (apart from $m_{\phi}=$ $50 \mathrm{GeV}$ which is near the decay threshold), whereas the reach in $\sin ^{2} \theta$ is larger for low $m_{\phi}$ - owing to the longer lifetime. While for $m_{\phi}=5 \mathrm{GeV}$ no natural parameter space is probed, for $m_{\phi}=10$ (15) GeV only FCCee (and HL-LHC) access the natural parameter space, and for higher masses this is also achieved in the present CMS analysis.

For the FCChh, the vast amount of produced Higgses can result in a very strong upper limit on the invisible branching ratio. Ref. [71] reports for a luminosity of $30 \mathrm{ab}^{-1}$ an expected sensitivity of a direct search to $\mathrm{BR}_{h \rightarrow \text { inv }} \lesssim 3 \cdot 10^{-4}$, i.e. similar to the result from a global fit of $\mathrm{BR}_{h \rightarrow \text { inv }} \leq 2.4 \cdot 10^{-4}$ [56]. The asymptotic limit on $\lambda_{h \phi}$ for vanishing $\sin ^{2} \theta$ can be approximated as

$$
\lambda_{h \phi}=\frac{2}{v} \sqrt{\frac{2 \pi m_{h} \Gamma_{\mathrm{tot}}^{\mathrm{SM}} \mathrm{BR}_{\mathrm{inv}}}{\sqrt{1-\frac{4 m_{\phi}^{2}}{m_{h}^{2}}}}} .
$$

This translates into the asymptotic bound on $\lambda_{h \phi}$ for $m_{\phi}=5 \mathrm{GeV}(50 \mathrm{GeV})$ of $\lambda_{h \phi} \leq$ $2.3 \cdot 10^{-4}\left(2.9 \cdot 10^{-4}\right)$ using the fit result, hence stronger than the limit of the direct searches for $h \rightarrow$ inv at the FCCee, and probing natural values of $\lambda_{h \phi}$ throughout this mass range. The approximate FCChh bounds are included in figures 8 and 9 up to values of $\sin ^{2} \theta$ for which all singlets can be safely assumed to decay outside the detector.

\section{Overview}

Having presented details about each search strategy in the previous section, here we compile them for comparison, to highlight their complementarity and to evaluate the probed parameter regions, both for the general singlet and the relaxion.

In figure 8 we show the coupling parameter plane spanned by $\sin ^{2} \theta$ and $\lambda_{h \phi}$ for benchmark values of $m_{\phi}=\{5,25,50\} \mathrm{GeV}$. For the singlet, the upper bound on $\lambda_{h \phi}$ from 


\begin{tabular}{|l|c|c|c|c|}
\hline Collider & $\sqrt{s}[\mathrm{TeV}]$ & $\mathcal{L}_{\text {int }}\left[\mathrm{ab}^{-1}\right]$ & $\mathrm{BR}_{\text {inv }}[\%]$ & Ref. \\
\hline LHC2+LHC1 & $7,8,13$ & $0.005,0.020,0.036$ & 19 & {$[72]$} \\
HL-LHC & 14 & 3 & 2.5 & {$[73]$} \\
FCCee240 & 0.24 & 5 & 0.3 & {$[74,75]$} \\
\hline
\end{tabular}

Table 2. Analyses of invisible Higgs decays recast in this work to constrain the scalar singlet.

\begin{tabular}{|c|c|c|c|c|}
\hline Collider & $\sqrt{s}[\mathrm{TeV}]$ & process & selections & Ref. \\
\hline $\begin{array}{c}\text { LHC2 } \\
+ \\
\text { LHC1 }\end{array}$ & 13 & VBF & $\begin{array}{c}p_{T}^{j} \geq 80(40) \mathrm{GeV} \\
\left|\Delta \eta_{j j}\right| \geq 1 \\
\left|\Delta \phi_{j j}\right| \leq 1.5 \mathrm{rad} \\
\eta_{j 1} \eta_{j 2} \leq 0 \\
\min \left|\Delta \phi\left(p_{T}^{j}, p_{T}^{\mathrm{miss}}\right)\right| \geq 0.5 \mathrm{rad} \\
E_{T}^{\mathrm{miss}} \geq 250 \mathrm{GeV} \\
m_{j j} \geq 200 \mathrm{GeV}\end{array}$ & {$[72]$} \\
\hline HL-LHC & 14 & $\mathrm{VBF}$ & $\begin{array}{c}p_{T}^{j} \geq 80(40) \mathrm{GeV} \\
\left|\Delta \eta_{j j}\right| \geq 4 \\
\left|\Delta \phi_{j j}\right| \leq 1.8 \mathrm{rad} \\
\min \left|\Delta \phi\left(p_{T}^{j}, p_{T}^{\mathrm{miss}}\right)\right| \geq 0.5 \mathrm{rad} \\
E_{T}^{\mathrm{miss}} \geq 190 \mathrm{GeV} \\
m_{j j} \geq 2500 \mathrm{GeV}\end{array}$ & {$[73]$} \\
\hline FCCee240 & 0.24 & $\begin{array}{c}\text { Higgs-strahlung: } \\
\quad e^{+} e^{-} \rightarrow Z h \\
Z \rightarrow \ell^{+} \ell^{-}, h \rightarrow \phi \phi\end{array}$ & $\begin{array}{c}p_{T}^{\ell}, p_{T}^{\ell \ell} \geq 10 \mathrm{GeV} \\
p_{L}^{\ell \ell} \leq 50 \mathrm{GeV} \\
\left|m_{\ell \ell}-M_{Z}\right| \leq 4 \mathrm{GeV}\end{array}$ & {$[74,75]$} \\
\hline
\end{tabular}

Table 3. Signal processes and selection cuts applied in the calculation of the fraction $r$ of invisible signal events. The $p_{T}^{j}$ cuts refer to the leading (subleading) jet.

naturalness according to eq. (2.22) is shown as the dotted curve. The dotted vertical line represents the natural upper bound on $\sin ^{2} \theta$, see eq. (2.19). In contrast, for the relaxion, the accessible $\lambda_{h \phi}$ within the natural band of $\sin ^{2} \theta$ is confined to the dark blue line that extends to larger $\sin ^{2} \theta$ than in the renormalizable singlet model, see figure 1 .

For both models, $\lambda_{h \phi}$ only impacts the decay of the Higgs into a pair of singlets, i.e. the number of produced $\phi \mathrm{s}$, whereas $\sin \theta$ mainly determines their lifetime $\tau_{\phi}$, and only contributes to $\mathrm{BR}_{h \rightarrow \phi \phi}$ for high $\sin \theta$.

The bounds from direct searches for invisible Higgs decays form horizontal, almost mass-independent, asymptotes on $\lambda_{h \phi}$ for sufficiently small $\sin ^{2} \theta$, where a scalar of the considered mass is still long-lived. Around this mass-dependent endpoint, the limit quickly weakens into a vertical asymptote. Both the analyses of displaced vertices and the timing 
method probe several orders of magnitude of $\sin ^{2} \theta$. The reach in $\lambda_{h \phi}$ of the ATLAS DV search is the strongest for an intermediate mass of $m_{\phi}=25 \mathrm{GeV}$, and relatively massindependent at the FCCee, whereas the timing bounds become stronger for higher masses. Here we show the bounds on the untagged Higgs decays introduced in section 3.1 only for large enough values of $\sin ^{2} \theta$, to ensure a decay within the detector. For smaller $\sin ^{2} \theta$, we show instead the (weaker) bounds on the additional Higgs width $\Gamma_{\mathrm{BSM}}=\Gamma(h \rightarrow \phi \phi)$, that are valid regardless of the decay length of $\phi$, hence down to arbitrarily low values of $\sin ^{2} \theta$. Because the specific decay of $\phi$ does not play a role, the shape is entirely determined by the $\lambda_{h \phi}$ and $\sin \theta$ contributions to the coupling $c_{h \phi \phi}$ in $\mathrm{BR}_{h \rightarrow \phi \phi}$. The green vertical lines represent the LEP1 bound [76] for the rare $Z \rightarrow \phi \ell \ell$ decay, and the GigaZ and TeraZ projections we obtained by rescaling with the ratio of produced $Z$ bosons, or the bound on $e^{+} e^{-} \rightarrow Z \phi$ at LEP2 [77] and ILC [64] which are stronger than the respective $Z$-decay constraint for $m_{\phi}=50 \mathrm{GeV}[32]$.

The natural parameter space of the general singlet with $m_{\phi}=5 \mathrm{GeV}$ has not been probed yet. Only small fractions of it can be probed by timing and displaced searches, as well as by fitting the untagged and BSM Higgs width and by searches of rare $Z$-decays. For the higher masses considered, all investigated bounds contribute to probing the natural parameter space, mainly because the upper naturalness bounds increase with the mass.

Considering the relaxion at $m_{\phi}=5 \mathrm{GeV}$, so far only the $Z$-decays at LEP1 marginally constrain the upper end of the natural region, which can be further probed by the same process at GigaZ, and excluded by TeraZ. Furthermore, untagged Higgs decays at future colliders are sensitive to the natural relaxion parameters. The heavier relaxion examples are already excluded by the BSM Higgs decays at the LHC1.

In figures 9 and 10 we show the bounds in the $m_{\phi}-\sin ^{2} \theta$ plane for the singlet scalar and for the relaxion, respectively. For the singlet scalar, we set the coupling $\lambda_{h \phi}=m_{\phi}^{2} / v^{2}=$ $\hat{\lambda}_{h \phi}^{\max }$, hence $\lambda_{h \phi}$ could be even larger. For the relaxion, the value of $\lambda_{h \phi}$ is given by eq. (2.33). In addition to the bounds discussed above, we also show the direct bound for $m_{\phi}<5 \mathrm{GeV}$ from $B \rightarrow K \mu \mu$ at the $\operatorname{LHCb}[19,78,79]$. Furthermore, we translate the uncertainties $\delta \kappa$ of the Higgs coupling modifier in global fits ${ }^{11}$ into model-independent bounds on $\sin ^{2} \theta$ that are independent of $m_{\phi}$ and $\lambda_{h \phi}$. The strongest bound stems from $\delta \kappa_{Z}$ at the FCChh (see table 1), and is shown in figure 10, but omitted in figures 8 and 9. From figure 10 we see that relaxions heavier than $\sim 18 \mathrm{GeV}$ are already excluded by the current LHC bounds on BSM Higgs decays. Rare $Z$-decays from LEP1 probe parts of the natural parameter space of the relaxion for $m_{\phi} \gtrsim 5 \mathrm{GeV}$, but the bound from the BSM Higgs branching at the LHC Run-1 is stronger than this LEP1 bound for $m_{\phi} \gtrsim 15 \mathrm{GeV}$. The best bounds from untagged Higgs decays will come from the FCChh, and can exclude relaxions above $m_{\phi} \gtrsim 8 \mathrm{GeV}$. On top of that, TeraZ can exlude relaxions of $m_{\phi} \gtrsim 3 \mathrm{GeV}$.

\footnotetext{
${ }^{11}$ We obtain the approximate $95 \%$ CL bound on $\sin ^{2} \theta$ from the provided $68 \%$ CL bound on $\delta \kappa$ with $\kappa=1+\delta \kappa$ by $\sin ^{2} \theta^{(95)} \simeq 1-\left(1+r \delta \kappa^{(68)}\right)^{2}$ where $r=\sqrt{q_{n}^{(95)} / q_{n}^{(68)}}$, and $q_{n}$ are the respective quantiles of a $\chi^{2}$-distribution with $n$ parameters.
} 

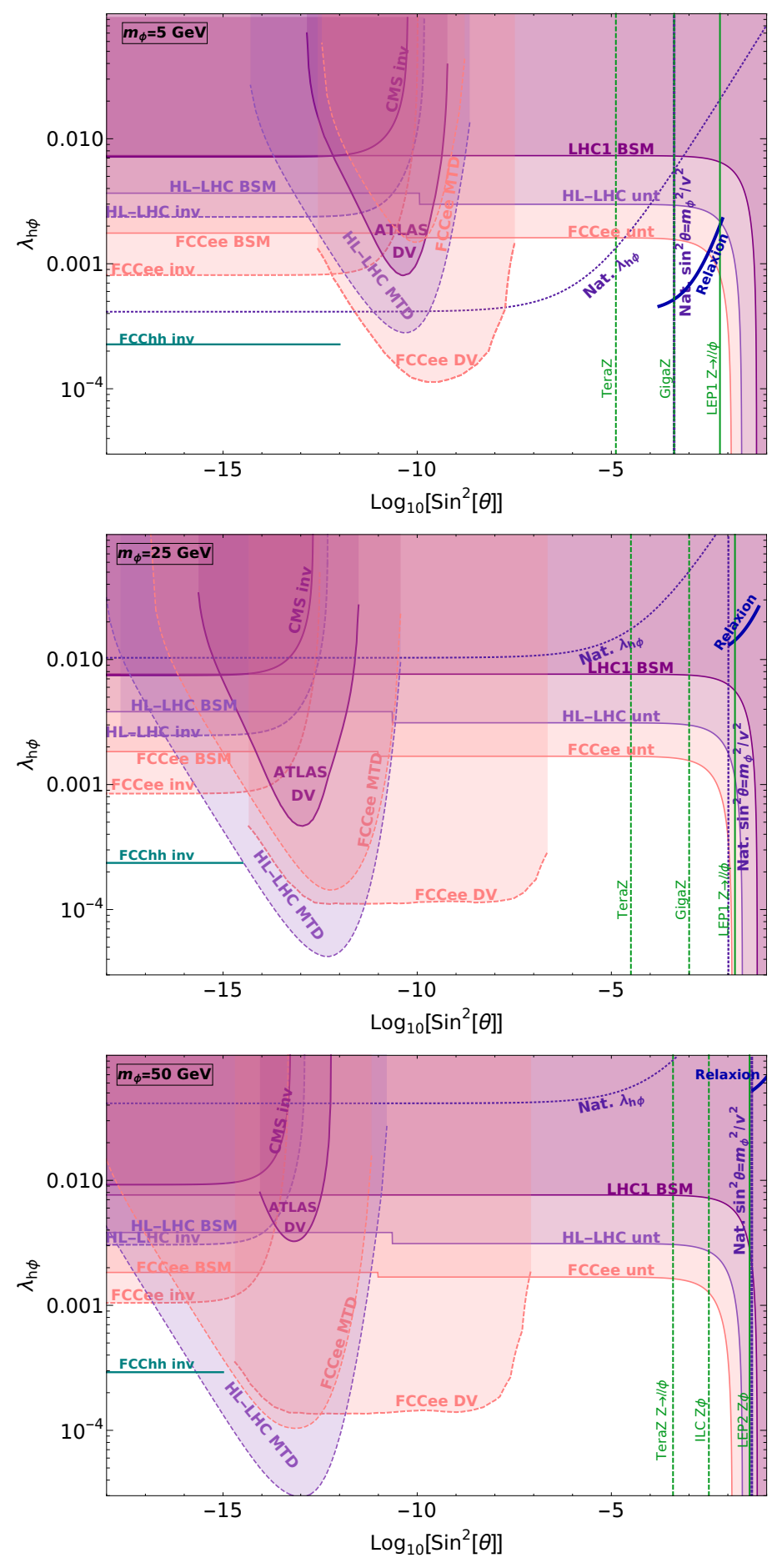

Figure 8. Direct and indirect bounds on $\lambda_{h \phi}$ and $\sin ^{2} \theta$ for the generic scalar singlet with a mass of $m_{\phi}=\{5,25,50\} \mathrm{GeV}$, respectively. The dotted lines represent the upper naturalness bounds on $\lambda_{h \phi}$ and $\sin \theta$ for the singlet. The thick blue lines represent the viable relaxion parameter space. In the $m_{\phi}=50 \mathrm{GeV}$ plot, we use the result of the ATLAS search for displaced jets [60] for $m_{\phi}=55 \mathrm{GeV}$. 


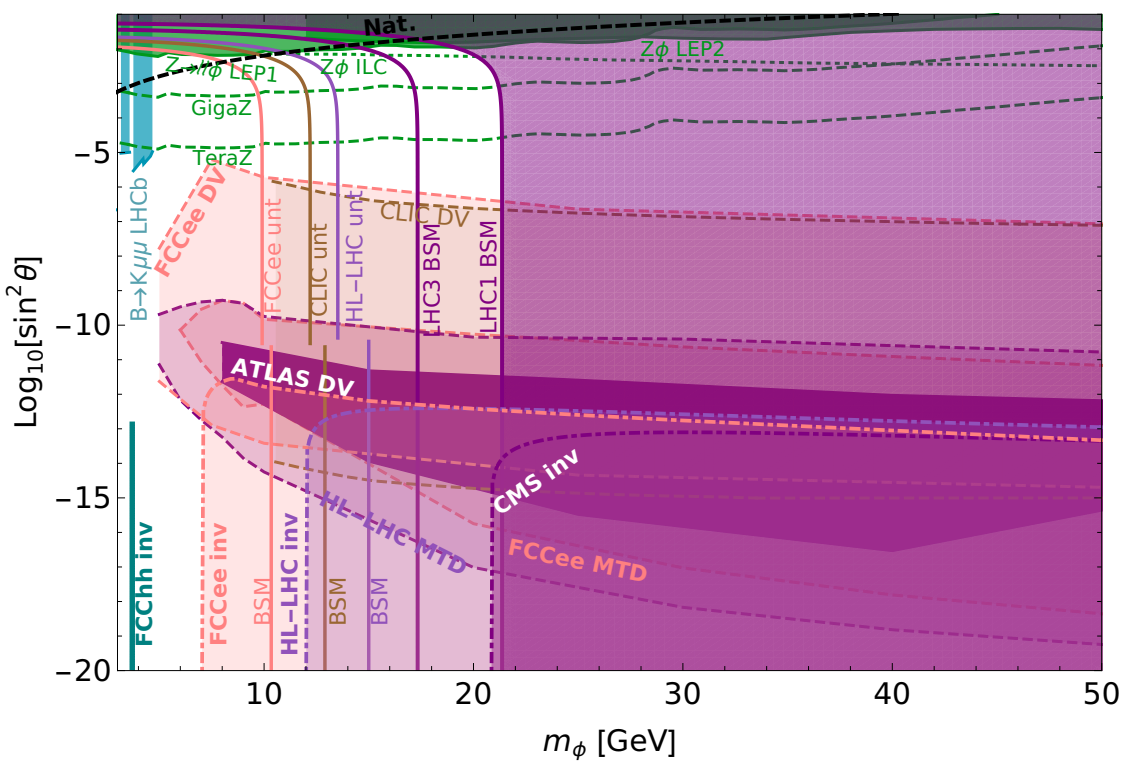

Figure 9. Bounds on $\sin ^{2} \theta$ and $m_{\phi}$ for the scalar singlet, with $\lambda_{h \phi}=m_{\phi}^{2} / v^{2}$ stemming from various hadron and lepton colliders and covering a large range of life times. The bounds labeled by BSM arise from the collider indicated by the untagged bound of the same color. For the bounds in the prompt region see also refs. [19, 32].

\section{Conclusions}

In this work, we exploit the sensitivity of the exotic Higgs decay channel $h \rightarrow \phi \phi$ to parameters of the relaxion and singlet models, taking into account existing searches and global fits, as well as projections for future colliders.

We discuss the renormalizable, non- $\mathbb{Z}_{2}$-symmetric singlet extension of the SM, focusing on the exotic Higgs decay $h \rightarrow \phi \phi$ via the triple scalar coupling $c_{h \phi \phi}$. The collider phenomenology is determined by the four parameters $m_{\phi}, \sin \theta, \hat{\lambda}_{h \phi}$, and $a_{\phi}$. Beyond the usual naturalness bound on the mixing angle, we present naturalness bounds on $\hat{\lambda}_{h \phi}$ and $a_{\phi}$ and investigate their implication on the physical parameter space. Moreover, we provide a matching between the singlet parameters and those of the relaxion. Here, the absence of a $\mathbb{Z}_{2}$ symmetry is pivotal to accommodate the linear slow-roll relaxion potential. The $h^{2} \phi^{2}$ term in the singlet model maps onto the first term of the expansion of the backreaction potential. We extend the naturalness relaxion band to higher masses relevant at colliders, where it is described by only two parameters, $m_{\phi}$ and $\sin \theta$, which determine $\lambda_{h \phi}$. Consequently, the relaxion model is both more constrained and predictive than the renormalizable singlet extension.

The lifetime of $\phi$, given by $\sin \theta$ and $m_{\phi}$, is the crucial handle in determining the kind of search strategy that sets the strongest bound. We study various lifetime dependent strategies. In particular, we evaluate the limits from global coupling fits on the new Higgs branching ratio into BSM, split into untagged and invisible final states; interpret the searches for the Higgs decaying into displaced jets in terms of the singlet model; exploit 


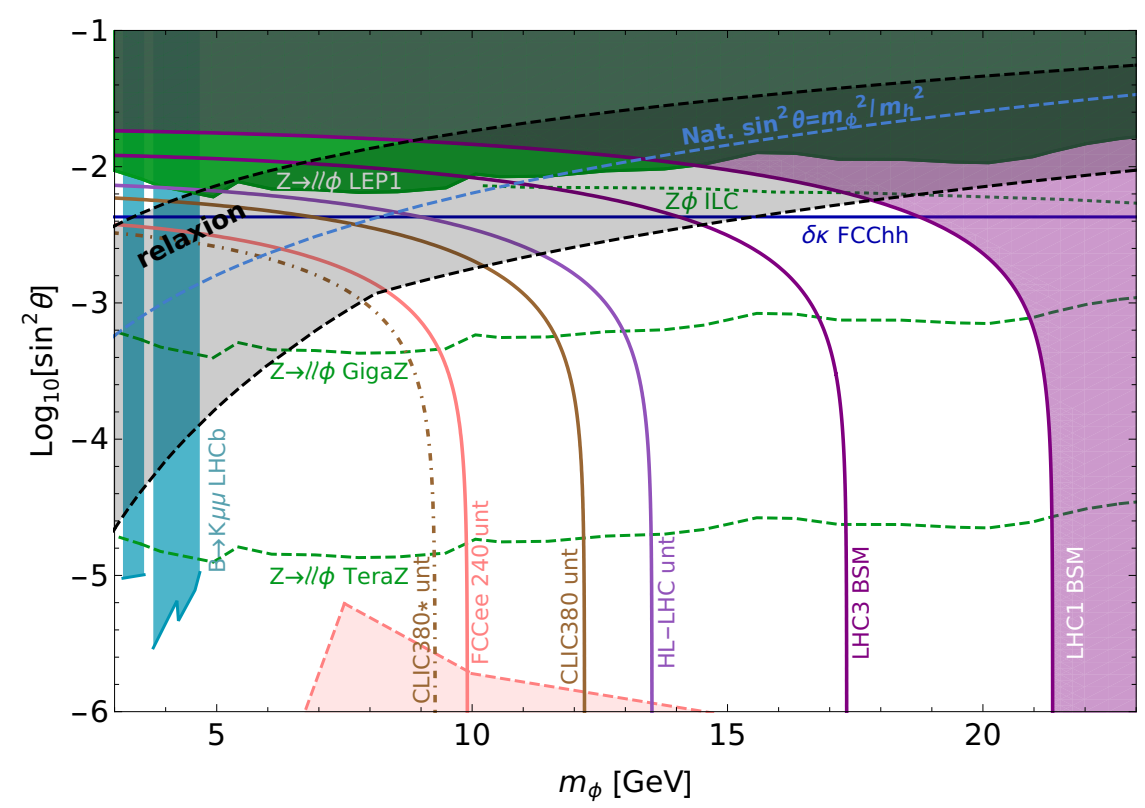

Figure 10. Prompt bounds on $\sin ^{2} \theta$ and $m_{\phi}$ for the relaxion, arising from direct and indirect probes at various hadron and lepton colliders, as in figure 9. The dash-dotted line stems from the one-parameter fit for CLIC as detailed in section 3.1. The gray band marks the region where the relaxion can be natural, see section 2.2. The upper bound is given by eq. (2.38) for the relaxion stopping in the first minimum $(n=1)$. The lower bound for low $m_{\phi}$ is dominated by the stopping in a generic minimum $(n \gg 1)$, see eq. (2.42); for high $m_{\phi}$ by the solution for $n=1$, see eq. (2.39). The bands corresponds to a choice of $\Lambda_{\min }=\tilde{M}_{\max }=1 \mathrm{TeV}$.

the time delay of jets originating from the $\phi$ decay to derive bounds in the region of intermediate lifetime; constrain the region of low $\sin ^{2} \theta$ by searches for invisible Higgs decays and pay attention to the range where the decay lengths are of the detector size such that only a fraction of the particles actually gives rise to the invisible signature.

Our main phenomenological findings are:

- For $m_{\phi}=5 \mathrm{GeV}$, only a small fraction of the natural singlet parameter space can be probed. For higher masses, larger coupling values become natural and the LHC has already excluded parts of it.

- The FCC can probe almost the complete considered parameter region by combining TeraZ, FCCee and FCChh, unless $\lambda_{h \phi}$ is much smaller than used here.

- The natural range for relaxions heavier than $18 \mathrm{GeV}$ is already excluded by searches for untagged Higgs decays at the LHC. The FCCee has the potential to exclude relaxions down to $8 \mathrm{GeV}$ using the same strategy. Only the search for rare $Z$ decays at TeraZ will be able to exclude the full mass range for heavy relaxions with $m_{\phi}>3 \mathrm{GeV}$.

\section{Acknowledgments}

We thank Gilad Perez for the initial collaboration, many valuable discussions, and helpful comments on the draft. Moreover, we thank Zhen Liu for useful information on timing 
bounds, and Christophe Grojean for interesting discussions. E.F. acknowledges the support by the Minerva Foundation during the initial phase of this work. M.S. is supported by the Alexander von Humboldt Foundation and thanks Fermilab for hospitality. The work of O.M. is supported by the Foreign Postdoctoral Fellowship Program of the Israel Academy of Sciences and Humanities. I.S. is supported by a fellowship from the Ariane de Rothschild Women Doctoral Program.

\section{A Relaxion stopping point}

The backreaction and the slow-roll potentials are defined in eqs. (2.26) and (2.23), respectively. Here we only consider $j=2$. The relaxion stops its evolution at $\phi_{0} \equiv \theta_{0} f$, given by

$$
V_{\mathrm{br}}^{\prime}\left(\theta_{0}\right)=-V_{\mathrm{sr}}^{\prime} \quad \Longrightarrow \quad \frac{v^{2}\left(\phi_{0}\right) \tilde{M}^{2}}{2 f} \sin \theta_{0}=-g \Lambda^{3} .
$$

In the following we set $r=1$ for simplicity, given that the exact expression for the relaxion mass only mildly depends on $r$. As the relaxion rolls down its potential before stopping, during each relaxation period $\Delta \phi=2 \pi f$ the maximal (in absolute value) slope of the oscillatory potential $V_{\mathrm{br}}^{\prime}$ changes by

$$
\Delta V_{\mathrm{br}}^{\prime} \simeq \frac{1}{2 f} \Delta v^{2} \tilde{M}^{2} \sin \theta_{\star} \simeq \frac{\pi}{\lambda_{h}} g \Lambda \tilde{M}^{2} \sin \theta_{\star} \simeq-\frac{\pi}{\lambda_{h} r} \frac{\tilde{M}^{2}}{\Lambda^{2}} \frac{v^{2}\left(\phi_{0}\right) \tilde{M}^{2}}{2 f} \sin \theta_{0} \sin \theta_{\star},
$$

where $\theta_{\star}=\phi_{\star} / f$ denotes the relaxion angle at which the $V_{\mathrm{br}}$ slope is maximized within the given $2 \pi f$ period, i.e. the inflection point of the periodic potential. $\Delta V_{\mathrm{br}}^{\prime}$ is $\tilde{M}^{2} / \Lambda^{2}$ suppressed with respect to the $V_{\mathrm{br}}^{\prime}$ overall size at the stopping point (A.1). Close to the final minimum, $\theta_{\star}$ can be found using eq. (2.32) for $m_{\phi}^{2} \simeq V^{\prime \prime}\left(\phi_{\star}\right)$, and solving

$$
V^{\prime \prime}\left(\phi_{\star}\right)=0 \Longrightarrow \frac{\cos \theta_{\star}}{\sin ^{2} \theta_{\star}}=\frac{\tilde{M}^{2}}{\lambda_{h} v^{2}\left(\phi_{\star}\right)},
$$

where $\lambda_{h} v^{2}\left(\phi_{\star}\right) \simeq \lambda_{h} v^{2}\left(\phi_{0}\right)+\tilde{M}^{2} \sin \theta_{\star}\left(\theta_{0}-\theta_{\star}\right)$ by a Taylor expansion of $\mu^{2}(\phi)$ neglecting the term suppressed by $g$. After the first minimum is formed, the slope of the periodic potential can overcompensate $V_{\mathrm{sr}}^{\prime}$ only by $\Delta V_{\mathrm{br}}^{\prime}$. After the $n$-th minimum it can do so by $n \Delta V_{\mathrm{br}}^{\prime}$. Correspondingly, the slope of the overall potential is given by the same value

$$
V^{\prime}\left(\phi_{\star}\right) \simeq n \Delta V_{\mathrm{br}}^{\prime} .
$$

We therefore know the position of the inflection point $\phi_{\star}$ and its slope $V^{\prime}\left(\phi_{\star}\right)$. They can be used to find the properties of the closest minimum $\phi_{0}$ located before $\phi_{\star}$. The value of $V^{\prime}\left(\phi_{0}\right)$ can be expressed as a Taylor series around $\phi_{\star}$

$$
0=V^{\prime}\left(\phi_{0}\right)=V^{\prime}\left(\phi_{\star}\right)+\frac{1}{2} V^{\prime \prime \prime}\left(\phi_{\star}\right)\left(\phi_{0}-\phi_{\star}\right)^{2}+\ldots
$$

with

$$
V^{\prime \prime \prime} \simeq-\frac{\tilde{M}^{2}}{2 f^{3}}\left(\frac{3}{2 \lambda_{h}} \tilde{M}^{2} \sin 2 \theta_{\star}+v^{2}\left(\phi_{\star}\right) \sin \theta_{\star}\right)
$$


Note that $V^{\prime \prime \prime}$ is obtained from the effective relaxion potential $V_{\text {eff }}$ after integrating out the Higgs boson, $h^{2} \rightarrow-\mu^{2}(\phi) / \lambda_{h}$, which is given by

$$
V_{\mathrm{eff}}=-\frac{1}{4 \lambda_{h}} \mu^{4}(\phi)+V(\phi)
$$

with $\mu^{2}\left(\phi_{0}\right)=-\lambda_{h} v^{2}$. Eq. (A.5) allows to find $\phi_{0}$ from

$$
\left(\phi_{0}-\phi_{\star}\right)^{2} \simeq-2 V^{\prime}\left(\phi_{\star}\right) / V^{\prime \prime \prime}\left(\phi_{\star}\right)
$$

and consequently all the related parameters of the theory. In particular, the relaxion mass can be approximated as

$$
m_{\phi}^{2}=V^{\prime \prime}\left(\phi_{0}\right) \simeq V^{\prime \prime \prime}\left(\phi_{\star}\right)\left(\phi_{0}-\phi_{\star}\right) \simeq \sqrt{\left|2 V^{\prime}\left(\phi_{\star}\right) V^{\prime \prime \prime}\left(\phi_{\star}\right)\right|}
$$

As we see, the mass is proportional to $\sqrt{V^{\prime}\left(\phi_{\star}\right)}$, which itself carries a factor $\tilde{M} / \Lambda$. This is precisely the reason why the relaxion mass is suppressed with respect to the naive estimate.

In this paper, we are interested in the corner of the parameter space where the relaxion reaches its maximal possible masses, which requires taking $\tilde{M} \gtrsim v$. In the limit $\tilde{M} \gg \sqrt{\lambda_{h}} v$, applicable within the relaxion mass range considered in this work, the relevant expressions simplify to

$$
\begin{aligned}
\theta_{0} & \simeq-\frac{\sqrt{\lambda_{h}} v}{\tilde{M}}+\sqrt{n} \sqrt{\frac{3 \pi \lambda_{h}^{1 / 2}}{2}} \frac{v^{3 / 2}}{\Lambda \tilde{M}^{1 / 2}}, \\
m_{\phi}^{2} & \simeq \sqrt{n} \sqrt{\frac{3 \pi}{2 \lambda_{h}^{1 / 2}} \frac{(v \tilde{M})^{5 / 2}}{f^{2} \Lambda}} .
\end{aligned}
$$

Inserting the relaxion angle $\theta_{0}$ into the general expression for the relaxion mass in eq. (2.32), we see that the small relaxion mass appears as a result of a fine cancellation between two contributions. Note that this also means that the loop corrections, otherwise subleading, may contribute sizeably to the relaxion mass. This, however, should not change qualitatively the results that we have derived, as the presence of the relaxion mass suppression is directly linked to the slow growth of the periodic barriers amplitude $-\Delta V_{\mathrm{br}}^{\prime} / V_{\mathrm{br}}^{\prime} \ll 1-$ the feature which is not expected to be altered by the loop effects.

For completeness we also write down corresponding expressions in the opposite limit, $\tilde{M} \ll v$, relevant for lighter relaxion, which were derived in ref. [31 $]^{12}$

$$
\begin{aligned}
\theta_{0} & \simeq-\pi / 2+\frac{\tilde{M}^{2}}{\lambda_{h} v^{2}}+\sqrt{n} \sqrt{\frac{2 \pi}{\lambda_{h}}} \frac{\tilde{M}}{\Lambda}, \\
m_{\phi}^{2} & \simeq \sqrt{n} \sqrt{\frac{\pi}{2 \lambda_{h}}} \frac{v^{2}}{f^{2} \Lambda} .
\end{aligned}
$$

\footnotetext{
${ }^{12}$ The reversed sign of $\theta_{0}$ is a consequence of a different sign convention for the relaxion potential.
} 


\section{B Estimating singlet production via Higgs mixing}

For small values of the coupling $\lambda_{h \phi} \ll \mathrm{s}_{\theta}^{2} m_{h}^{2} /\left(2 v^{2}\right)$, the branching ratio $\mathrm{BR}_{h \rightarrow \phi \phi}$ is proportional to $\sin ^{4} \theta$, cf. eq. (2.17). If in addition $\sin \theta$ is small, the Higgs almost never decays into a pair of scalars. On the other hand, the production of scalars via their mixing with the Higgs only scales as $\sin ^{2} \theta$ and becomes the dominant production mechanism if $\lambda_{h \phi}$ is small. However, if a sufficiently long lifetime is required in order to have a handle for the considered analyses, we estimate in the following that production via mixing yields only few events making a dedicated search difficult.

The number of scalars produced via mixing is given by $n_{\text {mix }}=\mathcal{L} \sigma_{\phi} \mathrm{s}_{\theta}^{2}$, where $\mathcal{L}$ is the luminosity and $\sigma_{\phi}$ is the production cross section of a Higgs boson with mass equal to $m_{\phi}$. Since detecting a dijet resonance at low mass is extremely challenging, we will consider only the searches for displaced jets or missing energy. To obtain a displaced or invisible signature, we need $c \tau \gtrsim 1 \mathrm{~cm}(\gtrsim 1 \mu \mathrm{m})$ for the HL-LHC (FCCee) which translates into $\sin ^{2} \theta \lesssim 10^{-9}\left(\lesssim 10^{-5}\right)$ using figure 2 for $m_{\phi}=5 \mathrm{GeV}$. A higher value for $m_{\phi}$ would be helpful in an analysis, but at the same time require even smaller mixing angles and also imply a smaller production cross section for kinematical reasons.

The HL-LHC will collect a luminosity of $\mathcal{L}=3 \cdot 10^{6} \mathrm{pb}^{-1}$. The production cross sections for a light Higgs at the LHC are below $100 \mathrm{pb}$ for all modes except for gluon fusion without $p_{T}$ requirement [32]. A leading order parton-level estimate with MadGraph5 for $\phi+j$ production at $14 \mathrm{TeV}$ with a very mild $p_{T}>20 \mathrm{GeV}$ requirement for the scalar yields $\sigma_{\phi} \approx 120 \mathrm{pb}$. Therefore the HL-LHC can only produce $n_{\text {mix }}^{\text {HL-LHC }} \lesssim 0.4$ non-prompt scalar singlets via mixing. Consequently, even before significant selection cuts no events will be available for an analysis.

The FCCee on the other hand will collect $\mathcal{L}=5 \cdot 10^{6} \mathrm{pb}^{-1}$ and the dominant production mode for a light Higgs at FCCee is Higgs-strahlung with a cross section of about $0.6 \mathrm{pb}$ [32] with $p_{T}^{\phi}>10 \mathrm{GeV}$. Therefore $n_{\text {mix }}^{\mathrm{FCCe}} \lesssim 30$. Considering more selective cuts on top of the minimal example cut applied here as well as the detector acceptance and e.g. leptonic $Z$ decay branching ratios, it will be impossible to have a sufficient number of scalars left for an analysis.

Here we argued why we focus only on $\phi$ production via Higgs decays. However, progress in detecting promptly decaying low-mass resonances may provide a new channel for singlet and relaxion searches [32]. Especially $b b, \tau \tau$ or $\mu \mu$ decays from production via mixing may allow to constrain the parameter regions where $\lambda_{h \phi}$ is negligible.

\section{Timing of delayed jets}

The crucial requirement of the analysis proposed in ref. [65] is that a jet leaving no track in the inner tracker hits the proposed timing layer with a delay $\Delta t>1 \mathrm{~ns}$ with respect to a (hypothetical) SM jet, going directly from the interaction point (IP) to the same location on the timing layer. This signature can be achieved by a particle that is invisible to the inner detector and decays into SM hadrons between the inner tracker and the timing layer. The delay is then a result both of the lower velocity of the heavier decaying particle, and of 

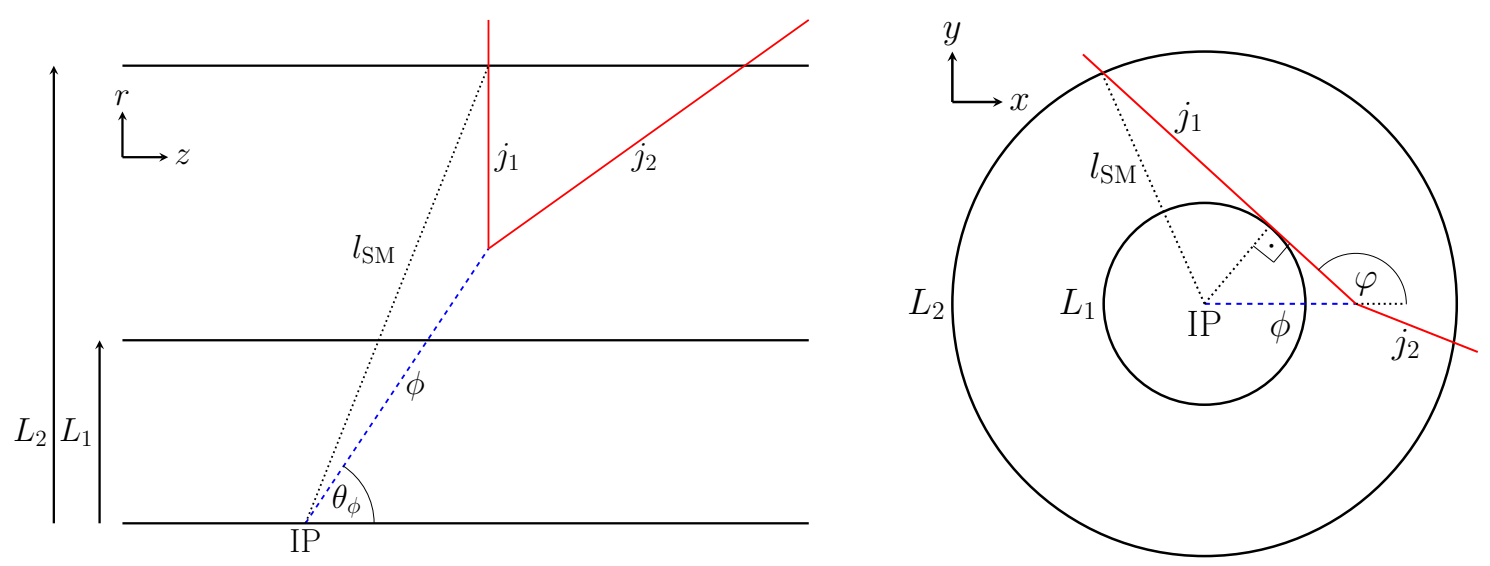

Figure 11. Sketch of the geometry in the longitudinal (left) and transverse (right) plane. Without loss of generality we show the case $\varphi_{\phi}=0$.

the displacement of the secondary decay in which the hadron is produced. For this reason, the acceptance probability of a given event is dominated by the geometrical trajectory of the decaying scalar and its decay product, once the kinematics is determined. Namely, once the four-momenta of the scalar and the jet are set, the lab-frame decay length of the scalar determines the secondary vertex position, the position in which the final jet hits the timing layer, and the overall time delay.

Since the analysis only requires at least one delayed jet, we can consider the four final state jets from the decay chain $h \rightarrow \phi \phi \rightarrow 4 j$ independently. Then, for a jet in a given event, we can find the range of allowed lab frame decay lengths of the scalar $l_{\phi, j}$, for which the jet will be accepted as signal. If this range is non-empty, we can assign a weight $w_{j}$, calculated as the probability to obtain $l_{\phi, j}$ within the allowed range, given that the proper decay length is $c \tau_{\phi}$, as in eq. (3.5). The probability for the whole event to be accepted is then given by $\epsilon_{\text {event }}=1-\left(1-w_{1}\right)\left(1-w_{2}\right)\left(1-w_{3}\right)\left(1-w_{4}\right)$.

In the following we will explain the computation of the allowed range of $l_{\phi, j}$. For the geometry in the detector, see figure 11. As described above, the scalar needs to decay between the outer radius of the inner tracker $L_{1}$ and the outer radius of the timing layer $L_{2}$. For CMS $L_{1}=0.2 \mathrm{~m}$ and $L_{2}=1.17 \mathrm{~m}$ [65], and for the FCCee we assume $L_{1}=0.127 \mathrm{~m}$ and $L_{2}=2.1 \mathrm{~m}[80]$. Thus, the distance the scalar may travel before decaying is constrained by $l^{L_{1}} \leq l_{\phi, j} \leq l^{L_{2}}$, given by

$$
l^{L_{1}}=\frac{L_{1}}{\sin \theta_{\phi}}, \quad l^{L_{2}}=\frac{L_{2}}{\sin \theta_{\phi}},
$$

where $\theta_{\phi}$ is the polar angle between the beam axis and the three-momentum of the considered scalar. In addition, we demand that the displaced jet does not cross the inner radius $L_{1}$ towards the beam axis, as it will leave a signature in the tracker. We thus require

$$
l_{\phi, j}^{\min }=\frac{L_{1}}{\sin \theta_{\phi}} \max \left(1,-\frac{\operatorname{sign}(\cos \varphi)}{|\sin \varphi|}\right),
$$


where $\varphi \equiv \varphi_{\phi}-\varphi_{j}$, and $\varphi_{\phi}$ and $\varphi_{j}$ refer to the azimuthal angles of the scalar and the jet, respectively.

The main selection criterion of the search is the time delay of the decay product, which is a result of the displaced vertex. The delay is defined as

$$
\Delta t=\frac{l_{\phi}}{c \beta_{\phi}}+\frac{l_{j}}{c \beta_{j}}-\frac{l_{\mathrm{SM}}}{c \beta_{\mathrm{SM}}}
$$

where $l_{\phi}$ is the distance traveled by the scalar before it decays, $l_{j}$ is the distance traveled by the decay product (a jet, in our case) to the timing layer, and $l_{\mathrm{SM}}$ is the distance a hypothetical SM particle would travel directly from the interaction point to the timing layer. The velocities of the particles are denoted by $\beta_{\phi}, \beta_{j}$ and $\beta_{\mathrm{SM}}$ in units of the speed of light $c$. Because the SM hadrons are light, $\beta_{\mathrm{SM}}=1$ to a good approximation. By demanding that the delayed jet hits the timing layer at radius $L_{2}$, and by setting $l_{\mathrm{SM}}=$ $\left|\vec{l}_{\phi}+\vec{l}_{j}\right|$, the time delay can be expressed solely as a function of the event kinematics and $l_{\phi}$. As the time delay has at most one maximum as a function of $l_{\phi}$, the allowed decay should lie between $l_{\max }^{\Delta t}, l_{\max }^{\Delta t}$, given by solving eq. (C.3) for $l_{\phi}$ with the required minimal time delay. Note that eq. (C.3) can be brought to a 4 th-degree polynomial form in $l_{\phi}$, and thus its roots can be found analytically. The temporal resolution of the timing layer is simulated by assigning normally distributed time stamps to the displaced jet hit $\delta_{j}$ and to the SM-ISR hit $\delta t_{\mathrm{ISR}}$, smeared by $\sigma=30 \mathrm{ps}$ [69], and requiring $\Delta t_{\mathrm{th}} \leq \Delta t+\delta t_{j}-\delta t_{\mathrm{ISR}}$, where $\Delta t_{\mathrm{th}}=1 \mathrm{~ns}$ is the minimal time delay set by the analysis.

Lastly, the decay product of the scalar should hit the timing layer at $L_{2}$ within the length of the detector (in the $\hat{z}$ direction), where we set $\left|z_{\max }\right|=2.6 \mathrm{~m}$ at CMS and $\left|z_{\max }\right|=2.3 \mathrm{~m}$ at the FCCee. If the scalar is produced at $z_{0}$, then the $z$ position of the hit of its decay product is

$$
Z \equiv l_{\phi} \cos \theta_{\phi}+l_{j} \cos \theta_{j}-z_{0}
$$

which is yet again completely determined by the event kinematics and $l_{\phi}$ (we set $z_{0}=0$ for simplicity, as the variations in the exact primary vertex position are negligible compared to the detector length). Therefore, imposing $-\left|z_{\max }\right| \leq Z \leq\left|z_{\max }\right|$ and solving for $l_{\phi}$ yields another set of constraints. Note that since $Z$ can have at most one extremum as a function of $l_{\phi}$, there may be at most two disconnected allowed ranges of $l_{\phi}$ satisfying the requirement above.

The final range of allowed decay lengths is then set by the union of the constraints given by the conditions above. For each allowed continuous range of $l_{\phi}, w$ is calculated by

$$
w_{j}=\exp \left(-\frac{l_{\phi, j}^{\min }}{c \tau_{\phi} \gamma_{\phi} \beta_{\phi}}\right)-\exp \left(-\frac{l_{\phi, j}^{\max }}{c \tau_{\phi} \gamma_{\phi} \beta_{\phi}}\right) .
$$

If the union has two or more disconnected regions, their contribution to $w$ should be summed. The resulting bounds on the Higgs branching to a pair of scalars and the efficiency of the search, both as a function of the lifetime, are presented in figure 12 . 


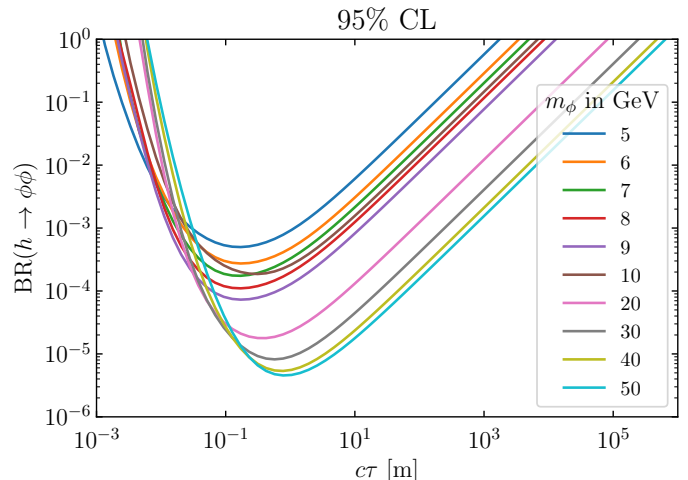

(a) HL-LHC $\sqrt{s}=14 \mathrm{TeV}$.

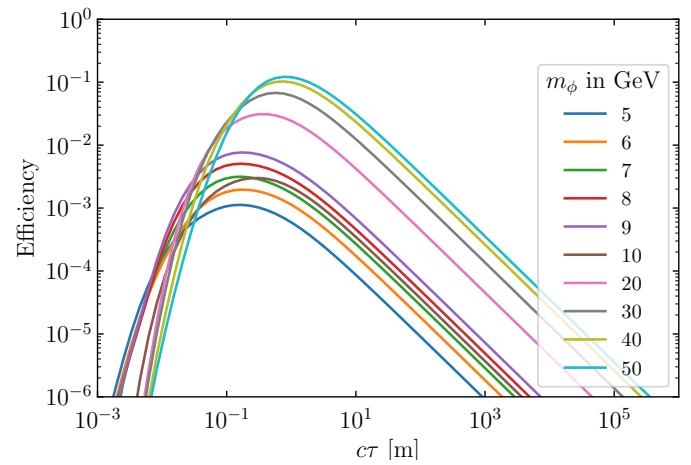

(c) HL-LHC $\sqrt{s}=14 \mathrm{TeV}$.

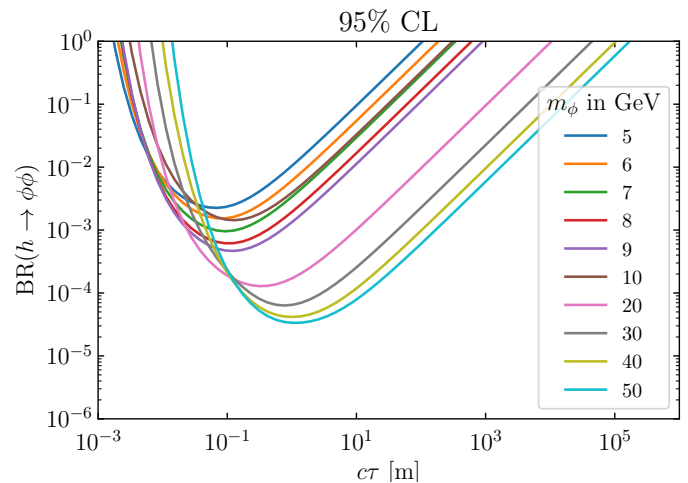

(b) FCC-ee $\sqrt{s}=240 \mathrm{GeV}$.

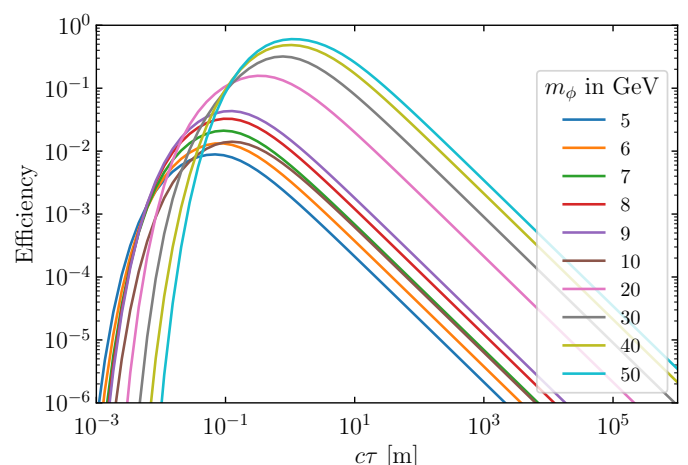

(d) FCC-ee $\sqrt{s}=240 \mathrm{GeV}$.

Figure 12. $\operatorname{BR}(h \rightarrow \phi \phi)$ and efficiency as a function of $c \tau_{\phi}$ for a search for delayed jets.

Open Access. This article is distributed under the terms of the Creative Commons Attribution License (CC-BY 4.0), which permits any use, distribution and reproduction in any medium, provided the original author(s) and source are credited.

\section{References}

[1] B. Patt and F. Wilczek, Higgs-field portal into hidden sectors, hep-ph/0605188 [InSPIRE].

[2] G.W. Anderson and L.J. Hall, The electroweak phase transition and baryogenesis, Phys. Rev. D 45 (1992) 2685 [INSPIRE].

[3] J.R. Espinosa and M. Quirós, The electroweak phase transition with a singlet, Phys. Lett. B 305 (1993) 98 [hep-ph/9301285] [INSPIRE].

[4] M. Carena, Z. Liu and Y. Wang, Electroweak phase transition with spontaneous $Z_{2}$-breaking, JHEP 08 (2020) 107 [arXiv: 1911.10206] [INSPIRE].

[5] J. Kozaczuk, M.J. Ramsey-Musolf and J. Shelton, Exotic Higgs boson decays and the electroweak phase transition, Phys. Rev. D 101 (2020) 115035 [arXiv:1911.10210] [INSPIRE].

[6] D. O'Connell, M.J. Ramsey-Musolf and M.B. Wise, Minimal Extension of the Standard Model Scalar Sector, Phys. Rev. D 75 (2007) 037701 [hep-ph/0611014] [inSPIRE].

[7] S. Adhikari, I.M. Lewis and M. Sullivan, Beyond the Standard Model Effective Field Theory: The Singlet Extended Standard Model, arXiv:2003.10449 [INSPIRE]. 
[8] D. Curtin et al., Exotic decays of the 125 GeV Higgs boson, Phys. Rev. D 90 (2014) 075004 [arXiv: 1312.4992] [INSPIRE].

[9] J.C. Berengut et al., Probing New Long-Range Interactions by Isotope Shift Spectroscopy, Phys. Rev. Lett. 120 (2018) 091801 [arXiv: 1704.05068] [InSPIRE].

[10] S. Aharony, N. Akerman, R. Ozeri, G. Perez, I. Savoray and R. Shaniv, Constraining Rapidly Oscillating Scalar Dark Matter Using Dynamic Decoupling, arXiv:1902.02788 [INSPIRE].

[11] D. Antypas, O. Tretiak, A. Garcon, R. Ozeri, G. Perez and D. Budker, Scalar dark matter in the radio-frequency band: atomic-spectroscopy search results, Phys. Rev. Lett. 123 (2019) 141102 [arXiv: 1905. 02968] [INSPIRE].

[12] J. McDonald, Gauge singlet scalars as cold dark matter, Phys. Rev. D 50 (1994) 3637 [hep-ph/0702143] [INSPIRE].

[13] C.P. Burgess, M. Pospelov and T. ter Veldhuis, The minimal model of nonbaryonic dark matter: A singlet scalar, Nucl. Phys. B 619 (2001) 709 [hep-ph/0011335] [InSPIRE].

[14] L.A. Ureña-López, Brief Review on Scalar Field Dark Matter Models, Front. Astron. Space Sci. 6 (2019) 47.

[15] A. Beniwal, M. Lewicki, J.D. Wells, M. White and A.G. Williams, Gravitational wave, collider and dark matter signals from a scalar singlet electroweak baryogenesis, JHEP 08 (2017) 108 [arXiv: 1702.06124] [InSPIRE].

[16] E. Hardy and R. Lasenby, Stellar cooling bounds on new light particles: plasma mixing effects, JHEP 02 (2017) 033 [arXiv: 1611.05852] [INSPIRE].

[17] J.D. Clarke, R. Foot and R.R. Volkas, Phenomenology of a very light scalar (100 MeV $<m_{h}<10 \mathrm{GeV}$ ) mixing with the SM Higgs, JHEP 02 (2014) 123 [arXiv:1310.8042] [INSPIRE].

[18] M.J. Dolan, F. Kahlhoefer, C. McCabe and K. Schmidt-Hoberg, A taste of dark matter: Flavour constraints on pseudoscalar mediators, JHEP 03 (2015) 171 [Erratum ibid. 07 (2015) 103] [arXiv:1412.5174] [INSPIRE].

[19] T. Flacke, C. Frugiuele, E. Fuchs, R.S. Gupta and G. Perez, Phenomenology of relaxion-Higgs mixing, JHEP 06 (2017) 050 [arXiv:1610.02025] [INSPIRE].

[20] K. Choi and S.H. Im, Constraints on Relaxion Windows, JHEP 12 (2016) 093 [arXiv: 1610.00680] [INSPIRE].

[21] V. Barger, P. Langacker, M. McCaskey, M.J. Ramsey-Musolf and G. Shaughnessy, LHC Phenomenology of an Extended Standard Model with a Real Scalar Singlet, Phys. Rev. D 77 (2008) 035005 [arXiv:0706.4311] [InSPIRE].

[22] T. Robens and T. Stefaniak, LHC Benchmark Scenarios for the Real Higgs Singlet Extension of the Standard Model, Eur. Phys. J. C 76 (2016) 268 [arXiv: 1601.07880] [inSPIRE].

[23] B. Heinemann and Y. Nir, The Higgs program and open questions in particle physics and cosmology, Usp. Fiz. Nauk 189 (2019) 985 [arXiv: 1905. 00382] [INSPIRE].

[24] P. Huang, A.J. Long and L.-T. Wang, Probing the Electroweak Phase Transition with Higgs Factories and Gravitational Waves, Phys. Rev. D 94 (2016) 075008 [arXiv:1608.06619] [INSPIRE].

[25] J. Beacham et al., Physics Beyond Colliders at CERN: Beyond the Standard Model Working Group Report, J. Phys. G $4 \mathbf{7}$ (2020) 010501 [arXiv: 1901.09966] [InSPIRE].

[26] P.W. Graham, D.E. Kaplan and S. Rajendran, Cosmological Relaxation of the Electroweak Scale, Phys. Rev. Lett. 115 (2015) 221801 [arXiv:1504.07551] [INSPIRE]. 
[27] N. Fonseca and E. Morgante, Relaxion Dark Matter, Phys. Rev. D 100 (2019) 055010 [arXiv: 1809.04534] [INSPIRE].

[28] A. Banerjee, H. Kim and G. Perez, Coherent relaxion dark matter, Phys. Rev. D 100 (2019) 115026 [arXiv: 1810.01889] [INSPIRE].

[29] R.S. Gupta, J.Y. Reiness and M. Spannowsky, All-in-one relaxion: A unified solution to five particle-physics puzzles, Phys. Rev. D 100 (2019) 055003 [arXiv:1902.08633] [INSPIRE].

[30] O. Davidi, R.S. Gupta, G. Perez, D. Redigolo and A. Shalit, The hierarchion, a relaxion addressing the Standard Model's hierarchies, JHEP 08 (2018) 153 [arXiv:1806.08791] [INSPIRE].

[31] A. Banerjee, H. Kim, O. Matsedonskyi, G. Perez and M.S. Safronova, Probing the Relaxed Relaxion at the Luminosity and Precision Frontiers, JHEP 07 (2020) 153 [arXiv: 2004.02899] [INSPIRE].

[32] C. Frugiuele, E. Fuchs, G. Perez and M. Schlaffer, Relaxion and light (pseudo)scalars at the HL-LHC and lepton colliders, JHEP 10 (2018) 151 [arXiv: 1807.10842] [INSPIRE].

[33] F. Piazza and M. Pospelov, Sub-eV scalar dark matter through the super-renormalizable Higgs portal, Phys. Rev. D 82 (2010) 043533 [arXiv:1003.2313] [InSPIRE].

[34] A. Ilnicka, T. Robens and T. Stefaniak, Constraining Extended Scalar Sectors at the LHC and beyond, Mod. Phys. Lett. A 33 (2018) 1830007 [arXiv: 1803.03594] [InSPIRE].

[35] S. Dawson and C.W. Murphy, Standard Model EFT and Extended Scalar Sectors, Phys. Rev. D 96 (2017) 015041 [arXiv: 1704.07851] [InSPIRE].

[36] A.V. Kotwal, M.J. Ramsey-Musolf, J.M. No and P. Winslow, Singlet-catalyzed electroweak phase transitions in the $100 \mathrm{TeV}$ frontier, Phys. Rev. D 94 (2016) 035022 [arXiv: 1605.06123] [INSPIRE].

[37] P.W. Graham, D.E. Kaplan, J. Mardon, S. Rajendran and W.A. Terrano, Dark Matter Direct Detection with Accelerometers, Phys. Rev. D 93 (2016) 075029 [arXiv:1512.06165] [INSPIRE].

[38] R.S. Gupta, Z. Komargodski, G. Perez and L. Ubaldi, Is the Relaxion an Axion?, JHEP 02 (2016) 166 [arXiv: 1509.00047] [INSPIRE].

[39] N. Fonseca, E. Morgante and G. Servant, Higgs relaxation after inflation, JHEP 10 (2018) 020 [arXiv: 1805. 04543] [INSPIRE].

[40] B. Batell, G.F. Giudice and M. McCullough, Natural Heavy Supersymmetry, JHEP 12 (2015) 162 [arXiv: 1509.00834] [INSPIRE].

[41] J.L. Evans, T. Gherghetta, N. Nagata and Z. Thomas, Naturalizing Supersymmetry with a Two-Field Relaxion Mechanism, JHEP 09 (2016) 150 [arXiv:1602.04812] [INSPIRE].

[42] B. Batell, M.A. Fedderke and L.-T. Wang, Relaxation of the Composite Higgs Little Hierarchy, JHEP 12 (2017) 139 [arXiv:1705.09666] [INSPIRE].

[43] K. Choi and S.H. Im, Realizing the relaxion from multiple axions and its UV completion with high scale supersymmetry, JHEP 01 (2016) 149 [arXiv:1511.00132] [INSPIRE].

[44] D.E. Kaplan and R. Rattazzi, Large field excursions and approximate discrete symmetries from a clockwork axion, Phys. Rev. D 93 (2016) 085007 [arXiv:1511.01827] [InSPIRE].

[45] A. Nelson and C. Prescod-WEinstein, Relaxion: A Landscape Without Anthropics, Phys. Rev. D 96 (2017) 113007 [arXiv:1708.00010] [InSPIRE].

[46] A. Hook and G. Marques-Tavares, Relaxation from particle production, JHEP 12 (2016) 101 [arXiv: 1607.01786] [INSPIRE]. 
[47] J.R. Espinosa, C. Grojean, G. Panico, A. Pomarol, O. Pujolàs and G. Servant, Cosmological Higgs-Axion Interplay for a Naturally Small Electroweak Scale, Phys. Rev. Lett. 115 (2015) 251803 [arXiv: 1506.09217] [INSPIRE].

[48] O. Davidi, R.S. Gupta, G. Perez, D. Redigolo and A. Shalit, Nelson-Barr relaxion, Phys. Rev. D 99 (2019) 035014 [arXiv: 1711.00858] [InSPIRE].

[49] N. Fonseca, E. Morgante, R. Sato and G. Servant, Axion fragmentation, JHEP 04 (2020) 010 [arXiv: 1911.08472] [inSPIRE].

[50] N. Fonseca, E. Morgante, R. Sato and G. Servant, Relaxion Fluctuations (Self-stopping Relaxion) and Overview of Relaxion Stopping Mechanisms, JHEP 05 (2020) 080 [Erratum ibid. 01 (2021) 012] [arXiv: 1911.08473] [INSPIRE].

[51] P. Bechtle, S. Heinemeyer, O. Stål, T. Stefaniak and G. Weiglein, Probing the Standard Model with Higgs signal rates from the Tevatron, the LHC and a future ILC, JHEP 11 (2014) 039 [arXiv:1403.1582] [INSPIRE].

[52] CMS collaboration, Precise determination of the mass of the Higgs boson and tests of compatibility of its couplings with the standard model predictions using proton collisions at 7 and $8 \mathrm{TeV}$, Eur. Phys. J. C $\mathbf{7 5}$ (2015) 212 [arXiv: 1412.8662] [InSPIRE].

[53] J. de Blas et al., The CLIC potential for new physics, CERN Yellow Reports: Monographs, CERN, Geneva, (2018), [DOI].

[54] CEPC Study Group collaboration, CEPC Conceptual Design Report: Volume 2 Physics 8 Detector, arXiv:1811.10545 [INSPIRE].

[55] X. Cid Vidal et al., Report from Working Group 3: Beyond the Standard Model physics at the HL-LHC and HE-LHC, CERN Yellow Rep. Monogr. 7 (2019) 585 [arXiv:1812.07831] [INSPIRE].

[56] J. de Blas et al., Higgs Boson Studies at Future Particle Colliders, JHEP 01 (2020) 139 [arXiv: 1905. 03764] [INSPIRE].

[57] ATLAS collaboration, Combined measurements of Higgs boson production and decay using up to $80 \mathrm{fb}^{-1}$ of proton-proton collision data at $\sqrt{\mathrm{s}}=13 \mathrm{TeV}$ collected with the ATLAS experiment, Phys. Rev. D 101 (2020) 012002 [arXiv: 1909.02845] [INSPIRE].

[58] LHC Higgs Cross Section Working Group collaboration, Handbook of LHC Higgs Cross Sections: 3. Higgs Properties: Report of the LHC Higgs Cross Section Working Group, CERN Yellow Reports: Monographs, (2013), arXiv:1307.1347 [INSPIRE].

[59] ATLAS collaboration, Search for long-lived particles produced in pp collisions at $\sqrt{s}=13 \mathrm{TeV}$ that decay into displaced hadronic jets in the ATLAS muon spectrometer, Phys. Rev. D 99 (2019) 052005 [arXiv: 1811.07370] [INSPIRE].

[60] ATLAS collaboration, Search for long-lived neutral particles in pp collisions at $\sqrt{s}=13 \mathrm{TeV}$ that decay into displaced hadronic jets in the ATLAS calorimeter, Eur. Phys. J. C 79 (2019) 481 [arXiv: 1902.03094] [INSPIRE].

[61] ATLAS collaboration, Search for long-lived neutral particles produced in pp collisions at $\sqrt{s}=13 \mathrm{TeV}$ decaying into displaced hadronic jets in the ATLAS inner detector and muon spectrometer, Phys. Rev. D 101 (2020) 052013 [arXiv: 1911.12575] [INSPIRE].

[62] S. Alipour-Fard, N. Craig, M. Jiang and S. Koren, Long Live the Higgs Factory: Higgs Decays to Long-Lived Particles at Future Lepton Colliders, Chin. Phys. C 43 (2019) 053101 [arXiv: 1812.05588] [INSPIRE].

[63] K. Cheung, O. Fischer, Z.S. Wang and J. Zurita, Exotic Higgs decays into displaced jets at the LHeC, JHEP 02 (2021) 161 [arXiv:2008.09614] [INSPIRE]. 
[64] R.K. Ellis et al., Physics Briefing Book: Input for the European Strategy for Particle Physics Update 2020, arXiv:1910.11775 [INSPIRE].

[65] J. Liu, Z. Liu and L.-T. Wang, Enhancing Long-Lived Particles Searches at the LHC with Precision Timing Information, Phys. Rev. Lett. 122 (2019) 131801 [arXiv:1805.05957] [INSPIRE].

[66] J. Liu, Z. Liu, L.-T. Wang and X.-P. Wang, Enhancing Sensitivities to Long-lived Particles with High Granularity Calorimeters at the LHC, JHEP 11 (2020) 066 [arXiv:2005.10836] [INSPIRE].

[67] Y. Gershtein, CMS Hardware Track Trigger: New Opportunities for Long-Lived Particle Searches at the HL-LHC, Phys. Rev. D 96 (2017) 035027 [arXiv:1705.04321] [INSPIRE].

[68] B. Bhattacherjee, S. Mukherjee, R. Sengupta and P. Solanki, Triggering long-lived particles in HL-LHC and the challenges in the rst stage of the trigger system, JHEP 08 (2020) 141 [arXiv: 2003.03943] [INSPIRE].

[69] CMS collaboration, Technical proposal for a MIP timing detector in the CMS experiment phase 2 upgrade, Tech. Rep. CERN-LHCC-2017-027. LHCC-P-009, CERN, Geneva (2017).

[70] J. Alwall et al., The automated computation of tree-level and next-to-leading order differential cross sections, and their matching to parton shower simulations, JHEP 07 (2014) 079 [arXiv: 1405.0301] [INSPIRE].

[71] L. Borgonovi et al., Higgs measurements at FCC-hh, Tech. Rep. CERN-ACC-2018-0045, CERN, Geneva (2018).

[72] CMS collaboration, Search for invisible decays of a Higgs boson produced through vector boson fusion in proton-proton collisions at $\sqrt{s}=13$ TeV, Phys. Lett. B 793 (2019) 520 [arXiv: 1809.05937] [INSPIRE].

[73] M. Cepeda et al., Report from Working Group 2: Higgs Physics at the HL-LHC and HE-LHC, CERN Yellow Rep. Monogr. 7 (2018) 221.

[74] P. Azzi et al., Prospective Studies for LEP3 with the CMS Detector, arXiv:1208.1662 [INSPIRE].

[75] M. Benedikt et al., FCC-ee: The Lepton Collider: Future Circular Collider Conceptual Design Report Volume 2. Future Circular Collider, Tech. Rep. CERN-ACC-2018-0057. 2, CERN, Geneva (Dec, 2018), DOI.

[76] L3 collaboration, Search for neutral Higgs boson production through the process $e^{+} e^{-} \rightarrow Z^{*} H^{0}$, Phys. Lett. B 385 (1996) 454 [InSPIRE].

[77] ALEPH, DELPHI, L3, OPAL and LEP Working Group for Higgs Boson SEARChES collaborations, Search for neutral MSSM Higgs bosons at LEP, Eur. Phys. J. C 47 (2006) 547 [hep-ex/0602042] [INSPIRE].

[78] LHCb collaboration, Differential branching fraction and angular analysis of the $B^{+} \rightarrow K^{+} \mu^{+} \mu^{-}$decay, JHEP 02 (2013) 105 [arXiv: 1209.4284] [INSPIRE].

[79] LHCb collaboration, Search for hidden-sector bosons in $B^{0} \rightarrow K^{* 0} \mu^{+} \mu^{-}$decays, Phys. Rev. Lett. 115 (2015) 161802 [arXiv: 1508.04094] [INSPIRE].

[80] N. Bacchetta et al., CLD - A Detector Concept for the FCC-ee, arXiv:1911.12230 [INSPIRE]. 